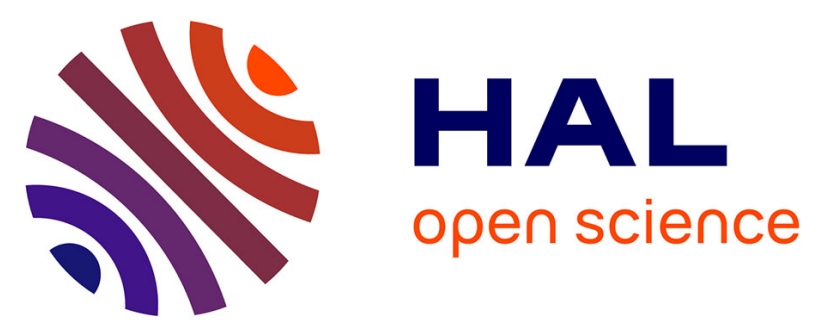

\title{
Measurement and modelling of aero-acoustic installation effects in tractor and pusher propeller architectures
}

\author{
Jernej Drofelnik, Matej Andrejašič, Blaž Močan, Tadej Kosel, Julien
}

Christophe, Joachim Dominique, Christophe Schram, Antoine Hajczak, Corneliu Stoica, Raluca Balasa, et al.

\section{To cite this version:}

Jernej Drofelnik, Matej Andrejašič, Blaž Močan, Tadej Kosel, Julien Christophe, et al.. Measurement and modelling of aero-acoustic installation effects in tractor and pusher propeller architectures. AIAA AVIATION 2021 FORUM, Aug 2021, VIRTUAL EVENT, United States. 10.2514/6.2021-2301 . hal03386210

\section{HAL Id: hal-03386210 https://hal.science/hal-03386210}

Submitted on 19 Oct 2021

HAL is a multi-disciplinary open access archive for the deposit and dissemination of scientific research documents, whether they are published or not. The documents may come from teaching and research institutions in France or abroad, or from public or private research centers.
L'archive ouverte pluridisciplinaire HAL, est destinée au dépôt et à la diffusion de documents scientifiques de niveau recherche, publiés ou non, émanant des établissements d'enseignement et de recherche français ou étrangers, des laboratoires publics ou privés. 


\title{
Measurement and modelling of aero-acoustic installation effects in tractor and pusher propeller architectures
}

\author{
Jernej Drofelnik *, Matej Andrejašič ${ }^{\dagger}$, Blaž Močan ${ }^{\ddagger}$ and Tadej Kosel ${ }^{\S}$ \\ Pipistrel Vertical Solutions d.o.o., Vipavska cesta 2, SI-5270 Ajdovščina, Slovenia \\ Julien Christophe II Joachim Dominique " and Christophe Schram ** \\ von Karman Institute for Fluid Dynamics, 1640 Rhode-St-Genèse, Belgium \\ Antoine Hajczak ${ }^{\dagger \dagger}$, \\ DAAA, ONERA, Université Paris Saclay, Châtillon F-92322, France

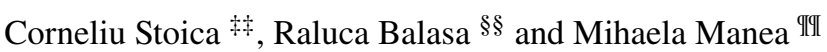 \\ INCAS - Institutul Național de Cercetare-Dezvoltare Aerospațială "Elie Carafoli" Bd. Iuliu Maniu nr. 220, București, \\ România
}

\begin{abstract}
Aero-acoustic installation effects are investigated both experimentally and numerically in the framework of the H2020 EC project ARTEM, for two Distributed Electric Propulsion configurations. In the tractor configuration, three propellers are installed on pylons protruding upstream of the leading-edge of a 1:3-scale realistic wing model. In the pusher configuration, the propellers are mounted on pylons extending from the suction side. Wind tunnel tests have been carried out in a closed-test section facility equipped with a microphone array, permitting to highlight the dominant aeroacoustic sources in both cases and providing validation data. In addition, the aero-propulsive performance has been assessed with balance measurements. The simulation strategies address both the tonal and broadband noise emissions due to the propeller and main wing self-noise, including scattering effects due to the wing and to the closed test section. These have been applied to a sub-selection of the wind tunnel test matrix that considers only one single propeller in both tractor and pusher configurations. The results show that the wind tunnel effects account for large part of the deviations observed between the free field noise predictions and the wind tunnel microphone measurements, but that the discrepancies can be partly compensated for by combining the aeroacoustic source models with Boundary Element and Finite Element acoustic simulations of the test section.
\end{abstract}

\section{Introduction}

The present work is inscribed in a research framework aimed at improving the understanding, modelling and mitigation of noise in distributed electric propulsion (DEP) configurations, through an ensemble of numerical and experimental activities. Electric and hybrid DEP solutions have become an increasingly attractive option for the aviation industry [1], and have been thoroughly studied in terms of aerodynamics, emissions and costs [2-5], but more scarcely investigated in terms of noise emissions. Previous efforts were mostly focused on the NASA X-57 [6, 7], or NASA GL-10 project [8, 9]. Several studies [6, 10,-12] indicate that DEP architectures offer promising perspectives for

\footnotetext{
*Research Engineer, Flight Physics Department

${ }^{\dagger}$ Head of Aerodynamics, Flight Physics Department

${ }^{\dagger}$ Head of R\&D, Research \& Development Department

$\S$ Test Engineer, Flight Physics Department

IISenior Research Engineer, Environmental and Applied Fluid Dynamics \& Aeronautics and Aerospace depts, 72 chaussée de Waterloo, AIAA Senior Member.

"PhD Candidate, Environmental and Applied Fluid Dynamics \& Aeronautics and Aerospace depts, 72 chaussée de Waterloo, AIAA Senior Member.

** Professor, Environmental and Applied Fluid Dynamics \& Aeronautics and Aerospace depts, 72 chaussée de Waterloo, AIAA Senior Member.

${ }^{\dagger}$ Research Engineer, Department of Aerodynamics, Aeroelasticity, Acoustics, Computational Aeroacoustics research unit

Head of Experimental Facilities Division

$\S$ Scientific Researcher, Technical Department

IIII Scientific Researcher, Technical Department
} 
noise reduction and appear as a viable solution for delivering the strict mid-to-long term environmental goals set by governmental agencies.

While this may a priori seem counter-intuitive if one considers the multiplicity of aerodynamic interactions taking place in a DEP system, which are generally synonymous of load fluctuations and noise production, several factors are actually in favor of noise reduction [12]:

1) Positive aerodynamic interactions such as wing blowing opens indirect perspectives for quieter transport. Besides, the efficiency of electric motors does not depend much on their size, so that the overall conversion efficiency of a large number of electric motors driving many small propellers can be as good as that of a single motor driving a single large propeller [13]. This means that either a smaller wing sized for cruise can be used, or that lower take-off and landing speeds are possible. The latter option is very promising in terms of noise emissions, since the aerodynamic noise radiated by the fixed parts of the airframe (landing gear and high-lift devices in particular) would typically radiate noise in proportion of the 5-6th power of the flight speed [14]. Moreover, the high lift-to-drag ratio allows steeper take-off and landing trajectories, which further reduces the noise footprint affecting communities.

2) The noise emitted by electric machines is much less than that associated to the compressor, combustor and turbine components. Huff et al. [15] reported that the noise generated by the electric motor system alone was 8-20 dB below the fan noise for a regional jet-sized aircraft, and 17-29 dB lower than that of a single-aisle commercial transport class aircraft.

3) While the proximity of the propulsive system with the airframe is normally a source of important tonal noise, the multiplicity of propulsors opens interesting perspectives for noise control via an adequate clocking of the propellers [6].

4) The flexibility offered by DEP systems permits placing the propulsors over the airframe in such a way to enhance noise shielding effects. Posey et al. [16] achieved low-frequency noise reductions of the order of $20 \mathrm{~dB}$ across a significant community area.

5) For turbo-electric distributed propulsion systems, i.e. when the electric power is provided by a common turbine, an effective by-pass ratio can be defined as the ratio of mass flow rate of all combined airflows by the one that enters the turbine. A high effective by-pass ratio is generally beneficial to noise reduction.

The main conclusion to be drawn from the above discussion, is that in order to properly address the noise reduction potential of DEP systems, all the relevant aspects should be tackled simultaneously: aero-propulsive benefits, noise shielding, tonal interference effects as well as non-aerodynamic noise sources. Naturally, the noise resulting from the unsteady aerodynamic interactions between the multiple propulsors and the airframe must not over-compensate the gains obtained from increased aero-propulsive efficiency. In this work, a simulation methodology was applied for the prediction of the noise produced by the rotor noise sources, namely steady loading, thickness, and trailing edge noise. The simulation results have been compared with experimental data obtained in the wind tunnel of INCAS.

The paper is structured as follows: the configurations of interest are presented in Sec. II the experimental set-up is described in Sec. III the simulation framework and the acoustic methods are detailed in Sec. IV] The results are given in Sec.V, and conclusions and perspectives are finally drawn in Sec.VI

\section{Propulsion architectures}

The proposed generic configurations are based on a scaling of a realistic DEP system designed by Pipistrel Vertical Solutions (PVS) for general aviation aircraft, with a chord of $1 \mathrm{~m}$ for a velocity of $40 \mathrm{~m} / \mathrm{s}$ in take-off conditions. This is deemed to be representative of the conditions causing annoyance to nearby communities. The considered DEP system itself has three bladed propellers with typical diameter of about $1 \mathrm{~m}$. The tip Mach number is about 0.55 and the Helmholtz number $\mathrm{He}$ based on the wing chord and the propeller blade passing frequency (BPF) is around 0.53. A scaling of approximately 1:3 with respect to the full-scale system has been chosen to respect the main similarity parameters while matching the possibilities of the INCAS wind tunnel described in Section III.A. Since all the relevant non-dimensional numbers $(\mathrm{Re}, \mathrm{Ma}, \mathrm{He})$ could not be preserved simultaneously, the priority was given to the tip Mach number and chordwise Helmholtz number in order to respect the important noise production and scattering mechanisms.

A propeller that fits the above criteria has been selected from reduced model manufacturers. A Master Airscrew clockwise propeller 13x8-inch has been selected, with a diameter of $0.33 \mathrm{~m}$. The considered wing has a PVS-DE(1)261717 profile of $0.3 \mathrm{~m}$ chord with maximum relative thickness of $17 \%$. The relevant parameters are described in Table 1 when considering the wind tunnel velocity of $30 \mathrm{~m} / \mathrm{s}$, as considered in the accompanying experiments.

Two different DEP implementations are considered, illustrated in Fig.11. a tractor configuration, where the propellers 
Table 1 Scaled model geometrical parameters.

\begin{tabular}{lcr} 
& Dimension & Value \\
\hline Wing chord & $\mathrm{m}$ & 0.3 \\
Free-stream velocity & $\mathrm{m} / \mathrm{s}$ & 30 \\
Propeller diameter & $\mathrm{m}$ & 0.33 \\
Number of blades & - & 3 \\
Propeller tip Mach number & - & 0.51 \\
Propeller RPM & rotations/minute & 10,000 \\
BPF & $\mathrm{Hz}$ & 500 \\
Wing chord-based Helmholtz number & - & 0.44 \\
\hline
\end{tabular}

are mounted on pylons protruding upstream of the wing leading edge, and a pusher configuration for which the propellers are supported downstream of a pylon mounted above the wing.

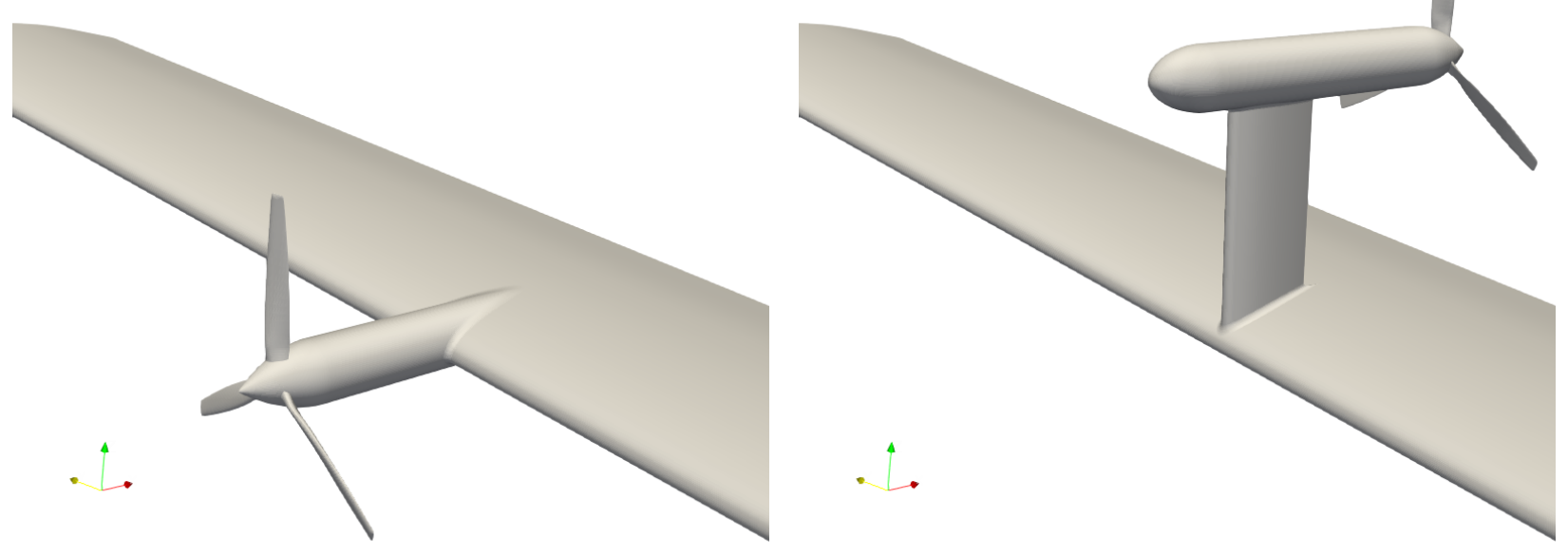

Fig. 1 (Left) Tractor and (right) pusher configurations.

Computational Fluid Dynamics (CFD) computations have been performed by PVS for the two configurations, in order to fine-tune the aerodynamic performance of both systems. Numeca Fine/Open solvers [17] were employed in this work. Aerodynamic performance was first assessed with the steady solver and actuator disk approximation, few points were then computed with unsteady solver using rotor/stator interfaces, to verify whether the trends obtained with actuator disk approximation approach were correct. In the case of the tractor configuration (Fig. 11 (left)), the aerodynamic optimum was found when the propeller is placed $0.2 \mathrm{~m}$ upstream of the wing leading-edge. For integration reasons of the engine, the propeller is placed slightly below the wing leading-edge. For the pusher configuration (Fig.11(right)), the propeller is placed at $0.181 \mathrm{~m}$ above the wing surface and $0.23 \mathrm{~m}$ downstream to the wing leading-edge, based on mechanical and aerodynamical considerations. In all the cases considered, the wing has an angle of attack of $5^{\circ}$. The final dimensions for both the tractor and pusher configurations are indicated in Fig. 3 (left). 


\section{Aerodynamic and aeroacoustic measurements}

\section{A. Wind Tunnel Specifications}

The INCAS Subsonic Wind Tunnel, used for this test campaign, is an atmospheric subsonic wind tunnel, single return type, closed-circuit, with a test section of $2.0 \mathrm{~m}$ high, $2.5 \mathrm{~m}$ wide and $4 \mathrm{~m}$ long. A 1.2 Megawatt engine is used to rotate a twelve blade propeller fan to move the air. The air speed is controlled by the operators by changing the RPM setting of the propeller. The maximum speed of this wind tunnel is $100 \mathrm{~m} / \mathrm{s}$ and the turbulence level factor is $\mathrm{K}=1.11$, as defined by Platt [18].

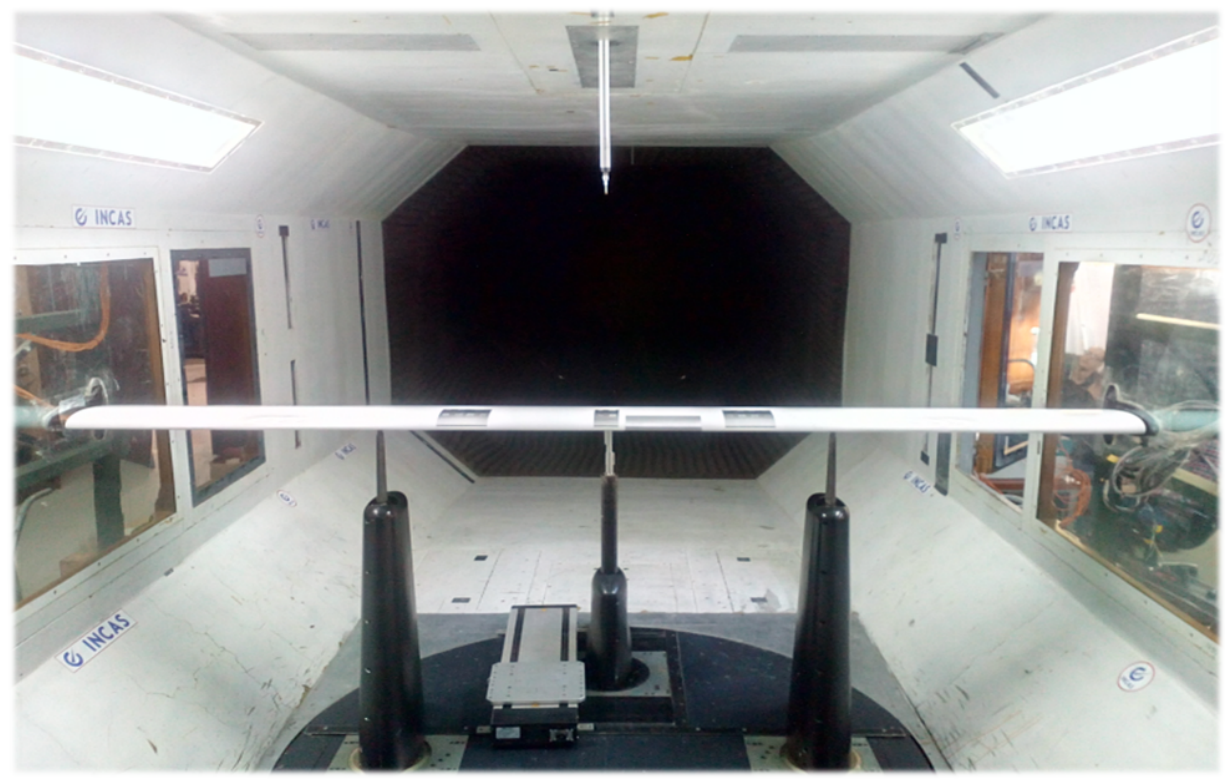

Fig. 2 Model mounted on aerodynamic struts within the INCAS wind tunnel for the aerodynamic force measurements.

For aerodynamic loads, the model is placed on the external balance struts, as shown in Fig. 2, the pitch and yaw angles are measured using optical encoders. It is also possible to fix the model from wall to wall and change the pitch angle by means of an external system based on electrical engines. The angles are set by the operators according to the test plan. After the angles are set the measurements are performed at fixed positions.

\section{B. Instrumentation}

The main data acquisition system is based on a computer capable of recording data signals but also controlling auxiliary PXI type chassis containing specialized data acquisition modules and other devices. The forces and moments are measured using a six-components external balance. The model is attached to the balance using special aerodynamically-shaped struts. The measurements using external balance are performed in step mode.

The pressure distribution on the wing was measured using pressure taps connected to two DSA 3217 scanners used in parallel. For this program, 32 pressure points, corresponding to each pressure tap on the wing are recorded. The recordings are made for every measurement point at indicated angle of attack ( 23 measurement points considering the model was moved from -7 to +15 degrees).

Regarding acoustic measurements, a circular array of diameter $1 \mathrm{~m}$, composed of 72 flush-mounted microphones with a $5^{\circ}$ angular discretization has been implemented in the ceiling of the wind tunnel for noise sources localization and characterization.

The wing is mounted in the wind tunnel side walls for the acoustic tests, in order to minimize the occurrence of spurious sources that would otherwise be caused by the struts, and upside-down (the pressure side on top). The external mounting system is actuated by two electrical engines that can provide wing adjustment at different incidence angles. The vertical distance between the wing quarter-chord line and the array is $0.845 \mathrm{~m}$, on the wing pressure side, while the distance between the wing trailing-edge and the array center is $0.06 \mathrm{~m}$. A summary of the mockup dimensions is 

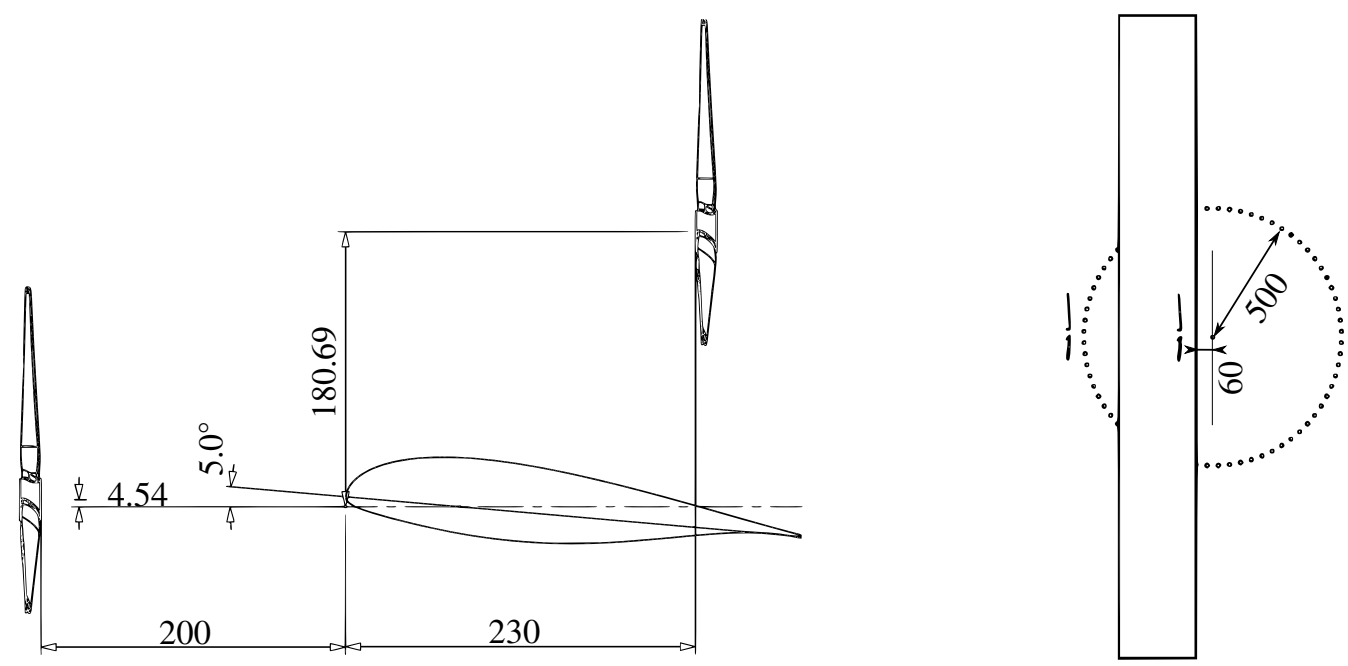

Fig. 3 Dimensions (in $\mathbf{m m}$ ) of the mockup and position of the microphone array relative to the wing.

presented in Fig. 3 .

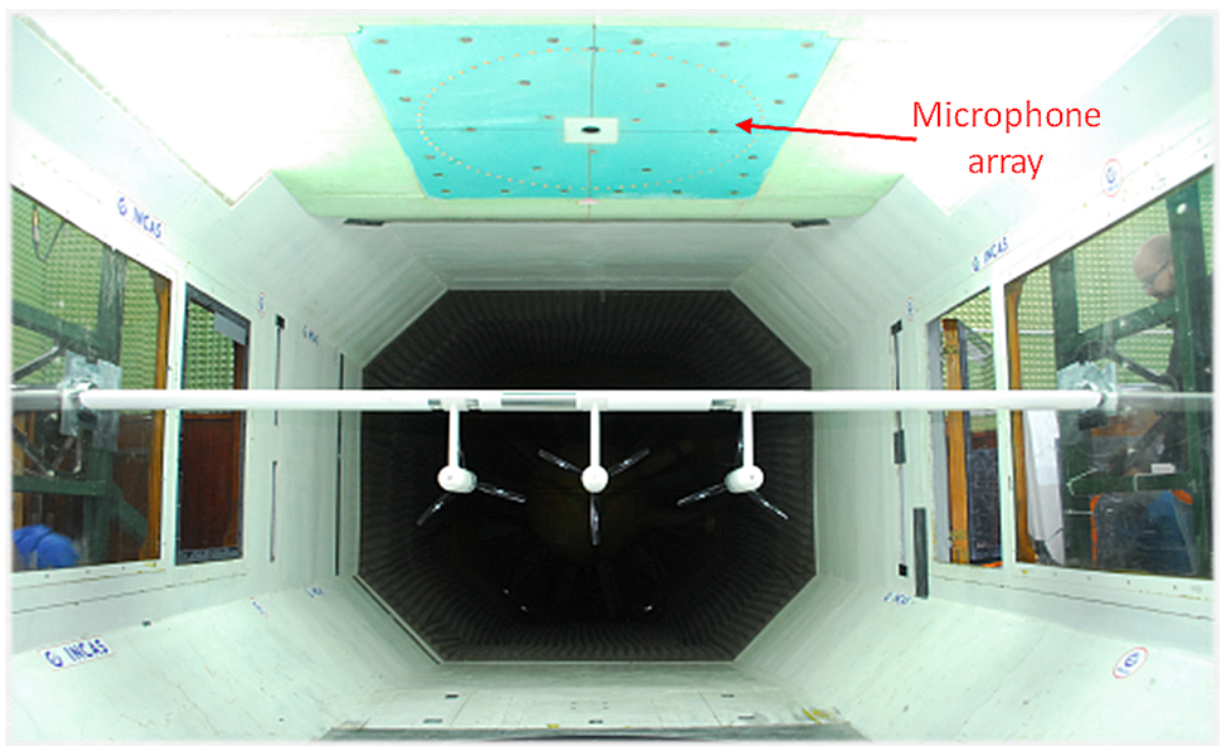

Fig. 4 Model mounted on the sidewalls of the INCAS wind tunnel for the acoustic measurements.

The industrial acquisition system contains signal conditioners and accurate, high resolution, 24-bit data acquisition boards. A video camera is mounted at the center of the microphones circle, enabling a fast localization of noise sources by overlapping the image of the viewed area and the noise "image" processed by the system. The signals from the microphones are first sent to the dedicated acquisition system that communicates with the main system via Ethernet gigabit connection. After recording, the data are processed using beamforming algorithm for source identification and acoustic "maps" are produced. Also, spectrum Fourier analysis is performed for each test case. For these tests each recording was for $10 \mathrm{~s}$ and the sampling frequency was $96 \mathrm{kHz}$.

Kulite sensors (XCQ-062) were installed at the wing leading edge, next to the middle propeller, in order to measure the wall pressure fluctuations induced by the propeller wakes impinging on the wing. Sensors are of differential type and can be used up to $20 \mathrm{kHz}$. The Kulite sensors signals are recorded by a second data acquisition system. The signal processing in this case consists in Fourier analysis.

The flow velocities in the propeller wake were measured using a hot wire constant temperature research grade 
anemometer provided by DANTEC. The device used is the Stream Line Pro, together with the Streamware Pro software. This equipment ensures a fast response in time and good sensitivity for this type of tests.

Each propulsion unit consists of Scorpion Turbax Conversion Kit w/ HK4035-800 motor, and Hobbywing FlyFun 110A HV V5 Brushless ESC SBEC electronic speed controller unit. Direct current laboratory power supply EA-PSI 9080-340 was used to power each propulsion unit, and U1210 Series 3.5 Digit Handheld Clamp Meter was used to measure the frequency, which can be directly converted to RPM setting.

\section{Test conditions}

Tests were performed for different model configurations, with varying parameters such as: flow speed, engines positions and numbers, propeller direction and different RPM settings. For all the aeroacoustic tests the model was kept at +5 degrees incidence angle so the flow was always aligned with the propeller axis. For the propulsion units in pusher configuration several cases were investigated by changing the number of propellers and their positions. For the aeroacoustic tests the wind tunnel speed was set at 20, 25, 30 and $35 \mathrm{~m} / \mathrm{s}$. For most of the tests $30 \mathrm{~m} / \mathrm{s}$ was used.

The runs are made using a range of propellers RPM settings. The main RPM used for aeroacoustic tests was 10,000 with slight variations. The other RPMs used were 6900, 7200, 8400, also with slight variations around these values. An aeroacoustic measurement was performed with the propellers free-spinning as well. Similarly, for propulsion units used in tractor configuration several cases were investigated by changing the propeller number, their positions and parameters.

For aerodynamic measurements, the balance external struts were put back in place and the model was mounted in normal position, enabling loads measurement. In this case the model is fixed in three points. The wing was in clean configuration, with no engine installed. The measurements were made at $30,40,50 \mathrm{~m} / \mathrm{s}$ wind speed and the incidence was changed from -7 to +15 degrees for each speed. During each measurement, the pressure distribution on the wing was measured. For better insights of the propeller wake hot wire measurements were done. The probe was moved along three traversing lines parallel to the propeller shaft. The measurements contain five data points for each of the measurement lines.

The results presented in this paper will focus on a sub-selection of the cases that have been tested in the wind tunnel. The measured aerodynamic coefficients for the wing alone will be presented in Sec. $\mathrm{V}$, along with the noise spectra in pusher and tractor configuration with only one propeller mounted on the wing.

\section{Noise mechanisms and simulation strategies}

A number of noise generation mechanisms are expected in both DEP configurations, listed in Fig. 5. At low Mach numbers and for frequencies low enough to make each source acoustically compact, noise production is intimately associated to the unsteady forces exerted by the solid surfaces on the surrounding fluid, either due to two-body interactions, or to mechanisms that are intrinsic to a given component.

The two-body aerodynamic interactions can be distinguished as viscous interaction and potential interaction [19]. The first instance corresponds to the wake of the propeller blade inducing unsteady forces on the wing in the tractor configuration, or the wake of the pylon being ingested by the propeller in the pusher case. It has been observed from CFD simulations that the velocity deficit of the propeller wake was already largely diffused at the location of the wing leading edge, leading the authors to leave the prediction of the associated noise aside in a first stage. Likewise, the interaction of the pylon wake with the propeller is left to further investigations. In any case, those viscous interaction mechanisms are associated to both tonal and broadband noise, due to the turbulence that is advected within the wake. Potential interaction induces tonal noise when the distance between the two involved components is small enough, typically of the order of couple of radii of curvature of the leading edge of the downstream element, which is not the case for the geometrical configurations considered here, and is therefore left as further work as well.

This paper is focused on the self-noise category, which includes the noise emitted by the turbulent boundary layer of the blade or wing when it reaches the trailing-edge [20, 21]. This mechanism is usually contributing to the broadband part of the sound spectrum, owing to the broad range of turbulent scales that are found in a fully developed turbulent boundary layer. In the case of propellers, two other self-noise mechanisms are due to the centrifugal acceleration of the steady forces, causing steady loading noise, and to the displacement of fluid by the blade motion, leading to the so-called thickness noise [22]. Both mechanisms, being periodic by nature, contribute to the tonal content of the noise spectrum, and be detailed in Section IV.A.

In theory, the complex unsteady phenomena that take place in DEP systems can be predicted via scale-resolved simulations such as Large Eddy Simulation [23], Detached Eddy Simulation [24] or Lattice Boltzmann Method [25]. However, even with modern computational resources, the numerical cost associated with such techniques would not be 


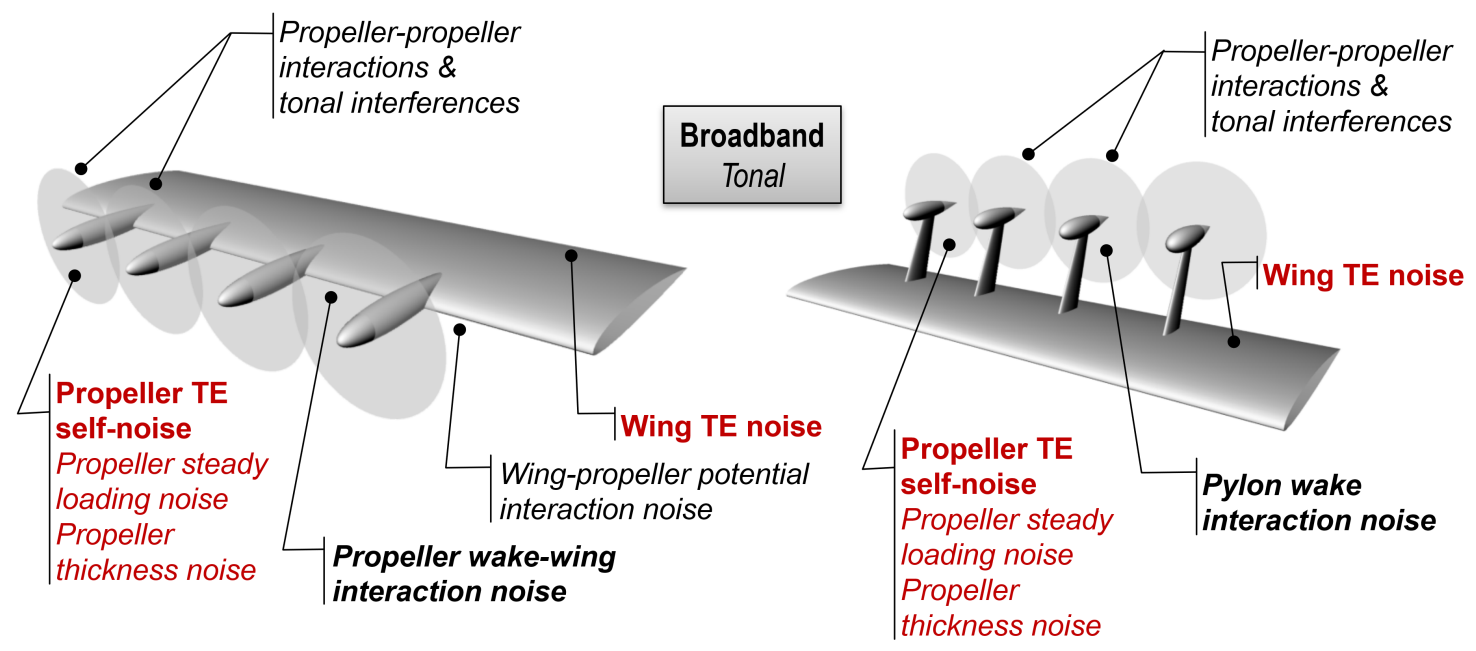

Fig. 5 Tonal (italic) and broadband (bold) noise generation mechanisms. Those indicated in red are modelled in the present study.

compatible with design and optimization requirements of a DEP system. For this reason, the modelling strategy is here rather based on mid-fidelity techniques such as (Unsteady) RANS simulations, more amenable to optimization runs at early design stages. The prediction of broadband self-noise is then achieved by means of semi-empirical models relating turbulence statistics to sound field statistics, as detailed in Section IV.B.

Beyond the aerodynamic installation effects, acoustic installation effects are also present when the noise is reflected by the airframe. This is in particular one of the beneficial effects that is sought with the pusher configuration, for which it is hoped that the wing will at least partly shield the listeners on the ground from the propeller noise. Analytical models have been derived to cope with simple geometries [19], such as the scattering of a propeller noise by the edge of a semi-infinite plane. In this work a more general and CPU-intensive approach has been followed, based on the numerical resolution of the wave propagation equation. It permits also taking into account the reflections of the wind tunnel test sections, as will be detailed below.

\section{A. Tonal self noise - Steady loading and thickness noise}

The thickness and steady loading noise contribute to the total sound field under the form of tonal components at the Blade Passing Frequency (BPF) harmonics. The thickness component corresponds to the periodic sound that results from the air displacement by the rotating blades. Therefore, it only depends on the blade geometry and kinematics and takes the form of a distribution of rotating monopole sources in the formalism of the Ffowcs Williams and Hawkings analogy [19, 26]. Steady loading noise corresponds to rotating dipole sources, of which amplitude reflects the mean aerodynamic loading of a compact blade surface element. In this work, this contribution has been assessed using ONERA's in-house mid-fidelity code PUMA. This code allows fast aerodynamic propeller performances predictions by combining a lifting-line approach with a free wake model based on Mudry's theory [27], which describes the unsteady evolution of a wake modeled by a potential discontinuity surface. The aerodynamic forces exerted on the propeller blades, obtained with the lifting-line method along the quarter-chord line at selected spanwise positions, are finally distributed along the chord using an empirical distribution model in order to obtain the wall pressure distribution on the blade surface required for the evaluation of the FW-H integral. Please note that, in the case of a propeller installed in the vicinity of a wing, PUMA is able to take into account the mutual interactions due to the respective induced velocities but is unable to simulate neither the wing or pylon potential effects, nor wake interaction viscous effects, which might actually not be negligible in DEP configurations. The inclusion of such effects in the aerodynamic loads prediction will be the subject of future work.

Both thickness and loading noise due to the propeller blades are then computed using ONERA's time-domain FW-H solver KIM [28], which provides, in addition to the free-field propeller noise in a uniform mean flow, the incident pressure on the possible scattering surfaces, along with the normal pressure gradient, both in complex form. Quadrupole noise, which becomes dominant only for transonic blade tip Mach numbers, has been neglected in this study (see Table 1), but it is important to mention that no far-field approximation is made in the FW-H solver, as the distance between the 
propeller and the wing and the acoustic wavelengths at the first BPF harmonics are of the same order of magnitude. More specifically, the formulation developed by Prieur \& Rahier [28] and implemented in KIM writes, for a uniform medium moving at $M$ in the $x$ direction:

$$
\begin{aligned}
p^{\prime}(\mathbf{x}, t)=\iint_{\Sigma}\left[\left(L_{i} \frac{\partial d}{\partial x_{i}}-Q U_{0} \frac{\partial d}{\partial x_{1}}\right) \frac{1}{4 \pi d^{2}|\partial g / \partial \tau|}\right]_{g=0} \mathrm{~d} S(\mathbf{y}) & \\
& +\frac{\partial}{\partial t} \iint_{\Sigma}\left[\left(L_{i} \frac{\partial g}{\partial x_{i}}-Q U_{0} \frac{\partial g}{\partial x_{1}}+Q\right) \frac{1}{4 \pi d|\partial g / \partial \tau|}\right]_{g=0} \mathrm{~d} S(\mathbf{y})
\end{aligned}
$$

where the Einstein summation convention is implicit and:

$$
L_{i}=p^{\prime} n_{i}+\rho u_{i}\left(u_{n}-v_{n}{ }^{\Sigma}\right), \text { and } Q=\rho_{0} v_{n}{ }^{\Sigma}+\rho\left(u_{n}-v_{n}{ }^{\Sigma}\right)
$$

The retarded-time function $g$ is:

$$
g=\tau-t+\frac{d-M\left(x_{1}-y_{1}\right)}{c_{0} \beta^{2}}
$$

And the distance $d$ is evaluated according to:

$$
d=\sqrt{C_{i}\left(x_{i}-y_{i}\right)^{2}} \text { where } C_{i}=\left(1-\beta^{2}\right) \delta_{1 i}+\beta^{2}
$$

Exact expressions for the derivatives $\partial d / \partial x_{i}, \partial g / \partial x_{i}, \partial g / \partial \tau$ can be found in Rahier et al. [29].

Finally, the total noise emitted by the installed propeller is obtained by performing a BEM computation with ONERA's solver BEMUSE [30]. The Brakhage-Werner formulation has been selected in the present work. BEMUSE benefits from several HPC developments based on H-matrix and Adaptive Cross Approximation algorithms that allow to run computations of the noise emitted by an installed propeller inside a wind tunnel with affordable computational resources. Please note that the current version of BEMUSE does not allow considering a background flow. However, considering the low free-stream Mach number in the experiments (see Table 1 , the assumption $M=0$ made during the computation is not expected to be a significant source of error.

\section{B. Broadband self noise - Trailing-edge noise}

The proposed approach for predicting the trailing-edge noise is based on semi-analytical models describing the unsteady loads induced over an element of wing or propeller and the propagation of the resulting equivalent dipoles [20, 31, 32]. The model includes compressibility and compactness effects [21], incoming turbulence distortion [33] and has been more recently extended to cope with trailing and leading-edge serrations for noise reduction [34, 35]. The key asset of the method is its rapidity, since predictions can be obtained in minutes for free field radiation problems, by using analytical or empirical wake models. A simulation platform has been developed by VKI based on that approach over the past decade, named BATMAN (Broadband And Tonal Models for Airfoil Noise).

The adopted methodology is shown in Fig. 6 The whole process is based on strip theory for which the propeller blades is divided in short segments along the spanwise direction, having their own radiated noise. The total emitted sound is then the summation of the noise produced by each of the strips. Following that approach, the blade is segmented following iso-radius cuts, for which the flow around the blade section can be obtained from two-dimensional or three-dimensional RANS computations. Using the 3D approach will allow to take into account the aerodynamic interaction between the different parts of the blade along the span compared to the $2 \mathrm{D}$ approach. From the flow around the blade iso-radius cuts, the trailing-edge boundary-layer profiles are extracted and wall-pressure models [36] are used to compute the wall-pressure spectrum near the trailing-edge. Together with geometrical blade parameters, this is the primary input to Amiet's theory [20] for trailing-edge noise. In order to take into account possible acoustic scattering by complex geometries, this theory can be combined with Acoustic Transfer Vector (ATV) calculated using numerical acoustic methods [37], as detailed below.

Solutions to both leading-edge and trailing-edge noise problems for thin airfoils have been proposed by Amiet [20, 31]. Amiet's solution considers chord-wise and span-wise distributed point dipoles where the far-field acoustic pressure generated by each point dipole is defined by Curle [38] as

$$
p\left(x, y, z, \omega ; x_{0}, y_{0}\right)=\frac{\mathrm{i} \omega z F\left(x_{0}, y_{0}\right) \mathrm{e}^{\mathrm{i} \omega t}}{4 \pi c_{0} \sigma_{t}^{2}} \mathrm{e}^{-\mathrm{i} \omega \sigma_{t} / c_{0}} .
$$




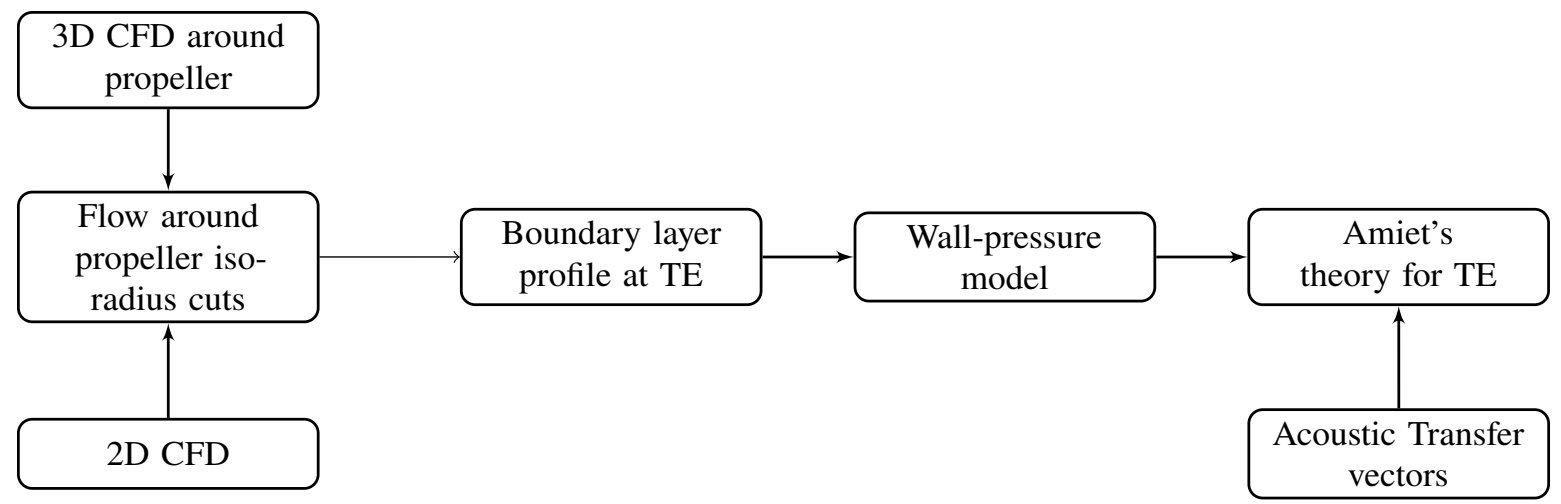

Fig. 6 Flowchart for propeller noise simulation.

The acoustic near-field terms have been assumed negligible for the high frequencies of interest. The observer-dipole distance is

$$
\sigma_{t}=\left(\sqrt{\left(x-x_{0}\right)^{2}+\beta^{2}\left(y-y_{0}\right)^{2}+\beta^{2} z^{2}}-M\left(x-x_{0}\right)\right) / \beta^{2}
$$

where $\left(x_{0}, y_{0}\right)$ are the chord-wise and span-wise positions of the point dipoles on the airfoil surface linearized as a flat plate with zero thickness, camber and angle of attack. $\omega$ and $c_{0}$ are the angular frequency and the speed of sound, respectively. $M$ is the Mach number of the uniform flow in the positive $x$ direction, and $\beta=\sqrt{1-M^{2}}$. $(x, y, z)$ are the coordinates of the observer. The origin is located at the center of the trailing edge of the airfoil.

The strength of the wall-normal dipole $F \mathrm{e}^{\mathrm{i} \omega t}$ was obtained from the unsteady pressure jumps of wall-pressure field considered as equivalent dipoles [20]. The formulation is derived analytically using an iterative application of Schwarzschild's solution [39].

The far-field solution to Amiet's trailing-edge noise model is computed as [20]

$$
S_{p p}(x, y, z, \omega)=\left(\frac{\omega b z}{2 \pi c_{0} \sigma^{2}}\right)^{2} \frac{1}{\pi} \int_{-\infty}^{\infty} \frac{\sin ^{2}\left[d\left(k_{y}-K_{y}\right)\right]}{\left(k_{y}-K_{y}\right)^{2}}\left|\boldsymbol{L}\left(x, K_{x}, k_{y}\right)\right|^{2} \Phi_{p p}(\omega) l_{y}\left(\omega, k_{y}\right) \mathrm{d} k_{y}
$$

where $\boldsymbol{L}\left(x, K_{x}, k_{y}\right)$ is the aeroacoustic transfer function, $\Phi_{p p}(\omega)$ the wall-pressure auto-spectrum directly extracted from a scale-resolved simulation and $l_{y}\left(\omega, k_{y}\right)$ the span-wise correlation length.

The current paper deals with the scattering of the emitted trailing-edge noise by surrounding surfaces, where the acoustic quantities need to be computed in the near-field of the airfoil making the geometrical far-field assumption questionable for scattering problems. A first-order geometrical near-field correction had already been proposed and validated for the leading-edge model [37]; the solution to Amiet's trailing-edge model then becomes

$$
S_{p p}(x, y, z, \omega)=\left(\frac{\omega b z}{2 \pi c_{0}}\right)^{2} \frac{1}{\pi} \int_{-\infty}^{\infty}\left|\boldsymbol{K}\left(x, y, z, K_{y}, k_{y}\right)\right|^{2}\left|\boldsymbol{L}\left(x, K_{x}, k_{y}\right)\right|^{2} \Phi_{p p}(\omega) l_{y}\left(\omega, k_{y}\right) \mathrm{d} k_{y}
$$

where the function $\boldsymbol{K}$ is expressed as a combination of exponential integrals $E_{1}$ [40]

$$
\begin{aligned}
\boldsymbol{K}\left(x, y, z, K_{y}, k_{y}\right)= & \frac{\mathrm{i} \mathrm{e}^{\mathrm{i}\left(K_{y}+k_{y}\right) y}}{2 \beta^{2} \sqrt{x^{2} / \beta^{2}+z^{2}}} \\
\{ & \mathrm{e}^{-\left(K_{y}+k_{y}\right) \sqrt{x^{2} / \beta^{2}+z^{2}}} E_{1}\left[-\left(K_{y}+k_{y}\right) \sqrt{x^{2} / \beta^{2}+z^{2}}-\mathrm{i}\left(K_{y}+k_{y}\right)(d-y)\right] \\
& -\mathrm{e}^{-\left(K_{y}+k_{y}\right) \sqrt{x^{2} / \beta^{2}+z^{2}}} E_{1}\left[-\left(K_{y}+k_{y}\right) \sqrt{x^{2} / \beta^{2}+z^{2}}-\mathrm{i}\left(K_{y}+k_{y}\right)(-d-y)\right] \\
& -\mathrm{e}^{\left(K_{y}+k_{y}\right) \sqrt{x^{2} / \beta^{2}+z^{2}}} E_{1}\left[\left(K_{y}+k_{y}\right) \sqrt{x^{2} / \beta^{2}+z^{2}}-\mathrm{i}\left(K_{y}+k_{y}\right)(d-y)\right] \\
& \left.+\mathrm{e}^{\left(K_{y}+k_{y}\right) \sqrt{x^{2} / \beta^{2}+z^{2}}} E_{1}\left[\left(K_{y}+k_{y}\right) \sqrt{x^{2} / \beta^{2}+z^{2}}-\mathrm{i}\left(K_{y}+k_{y}\right)(-d-y)\right]\right\}
\end{aligned}
$$


This generalization of Amiet's theory for trailing-edge noise has been validated on a stationary airfoil by Christophe $e t$ al. [41].

In case of rotation, the far-field noise PSD of a low solidity fan with $B$ independent blades is given by an integration over all possible azimuthal positions [42] of the single airfoil formulation (8)

$$
S_{p p}\left(\mathbf{X}, \omega_{f}\right)=\frac{B}{2 \pi} \int_{0}^{2 \pi}\left(\frac{\omega_{e}(\Psi)}{\omega_{f}}\right)^{2} S_{p p}^{\Psi}\left(\mathbf{x}, \omega_{e}\right) d \Psi
$$

where the factor $\omega_{e}(\Psi) / \omega_{f}$ accounts for Doppler effects caused by the relative motion between the blade and the observer due to rotation, $\omega_{e}$ being the emission angular frequency. Sinayoko et al. [43] have shown that this approximation of locally translating airfoils is accurate below transonic speeds.

Equation (8) assumes uniform flow profile along the span, which is not the case due to the rotation and possible installation effects. The fan noise predictions therefore rely on a strip theory in order to take into account the variation of the flow along the blade span. The latter is split in equal segments from hub to tip as demonstrated in Christophe $e t$ al. [44], and the total radiated sound is then the summation of the sound emitted by each blade strip.

In free-field, the sound is assumed to propagate from the source to the observer directly. However, in presence of surrounding surfaces, the sound generated is reflected and scattered by these surfaces. The scattering problem can be tackled by combining the free-field approaches described above with Boundary Element Method (BEM) or Finite Element Method (FEM) solutions of the Helmholtz equation [45, 46]. The present method is based on the decomposition of the acoustic pressure field into incident and scattered fields:

$$
p=p_{i}+p_{s}
$$

where the incident field pressure is $p_{i}$. In principle, the scattered pressure, $p_{s}$, can then be computed using a numerical acoustic model [45], with Neumann boundary conditions applying at the scattering surface. However, both BEM and FEM methods are deterministic, i.e. they require a description in amplitude and phase of the incident acoustic field, while Amiet's approach provides only a statistical model for the incident sound power spectrum (10). An alternative formulation has been proposed to circumvent this difficulty [47], based on the derivation of the total sound pressure power spectrum derived from (11):

$$
S_{p p}=S_{p_{i} p_{i}}+S_{p_{i} p_{s}}+S_{p_{s} p_{i}}+S_{p_{s} p_{s}}
$$

where the first term on the right-hand-side of the equation is the free-field response of the airfoil which can be obtained with Eq. [10]. The remaining terms involve the numerical acoustic BEM or FEM solvers of Simcenter 3D [45], by means of Acoustic Transfer Vectors (ATV) relating the acoustic pressure at an observer point to the wall-normal velocity of the elements that discretize the surface of the scattering body (here called the acoustic mesh):

$$
p_{s}(\omega)=\{\operatorname{ATV}(\omega)\} \cdot\left\{v_{n_{s}}(\omega)\right\}
$$

where $\{\operatorname{ATV}(\omega)\}$ is the Acoustic Transfer Vector matrices obtained by solving the BEM/FEM problem with unit normal velocities on the acoustic mesh [48]. The braces indicate matrix notation. In order to satisfy the Neumann boundary condition on the non-vibrating acoustic mesh [45], the velocities $\left\{v_{n_{s}}(\omega)\right\}$ in Equation [13] correspond to minus the projection of the incident velocity, $v_{n_{s}}=-v_{n_{i}}$. The incident velocity term, $v_{n_{i}}$, is computed from the gradient of the incident pressure:

$$
v_{n_{i}}(\mathbf{x}, \omega)=\frac{\mathrm{i}}{\rho_{0} \omega} \frac{\partial p(\mathbf{x}, \omega)}{\partial n} .
$$

Combining Eqs. (12) to 14, the total spectrum yields

$$
S_{p p}=S_{p p, i}-S_{p v} \cdot \mathbf{A} \mathbf{T V} \mathbf{V}^{T}-\mathbf{A} \mathbf{T V} \mathbf{V}^{*} \cdot S_{v p}+\mathbf{A} \mathbf{T V} \mathbf{V}^{*} \cdot S_{v v} \cdot \mathbf{A} \mathbf{T V} \mathbf{V}^{T}
$$

where the superscript ${ }^{T}$ stands for the transpose-matrix operator. $S_{p v}$ and $S_{v p}$ are the cross-power spectra of the incident velocity evaluated over the acoustic mesh and the incident acoustic pressure at the observer point. The $S_{v v}$ is the auto and cross-power spectra of the incident velocities over the acoustic mesh. The reader is referred to related reference [49] for the detailed derivation of cross-power spectra. The $\{\mathbf{A T V}\}$ is now a matrix composed of the ATVs for each observer and acoustic mesh points. They have been computed using the FEM solver of the Simcenter 3D simulation platform [45], featuring Adaptative Order finite elements (FEM-AO, [50, 51]) and Automatic Matched Layers (AML, [52]) for non-reflective boundary conditions. 


\section{Results}

\section{A. Aerodynamic and aeroacoustic measurements}

The acoustic post-processing is made using 10 seconds of signal recorded by the 72 microphones on the array. To highlight only the essential signal and remove the wind tunnel self-noise, a band pass filter is applied with lower and upper cutoff frequencies of $315 \mathrm{~Hz}$ and $20 \mathrm{kHz}$, respectively. At 10,000 RPM, the main rotation speed used for these tests, the propellers blade-passing frequency is $500 \mathrm{~Hz}$. For each run of this type, a frequency spectrum is produced as the average of the signals coming from all microphones. Additionally, the spectrum with the airfoil in "clean" configuration (no propeller mounted on) has been computed for direct comparison with the other measurements. In most cases, the noise emitted by the propellers emerges notably at the blade passing frequency harmonics (see Fig. 7 , where the gray columns represent third octave band levels).

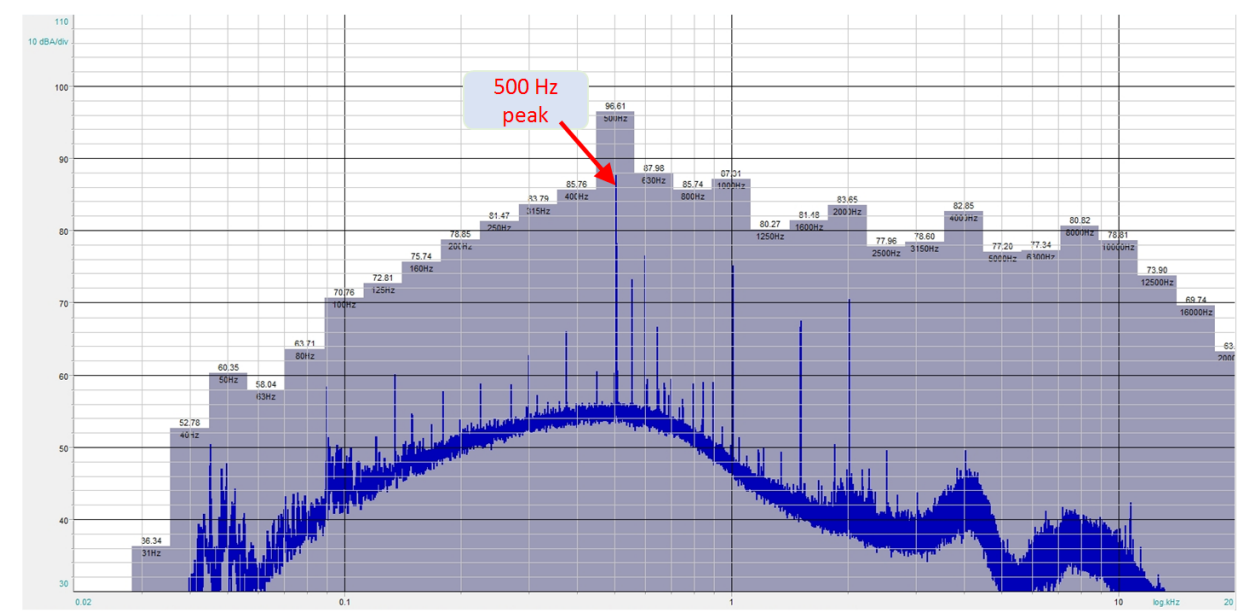

Fig. 7 Frequency spectrum (propeller noise peaks identification).

In order to localize the noise sources, a beamforming algorithm is used in the analyzed area. The video camera, located in the middle of the microphone array, produces a picture which the beamforming results are subsequently combined with. In this way the pictures colors show the corresponding pressure levels of the noise sources inside the targeted area. The pictures are expected to show the noise sources which are the highest in amplitude, but in some cases, the reflections from the walls or parts of the wing make the interpretation of the noise maps challenging. An exemple of such analysis is shown in Fig. 8

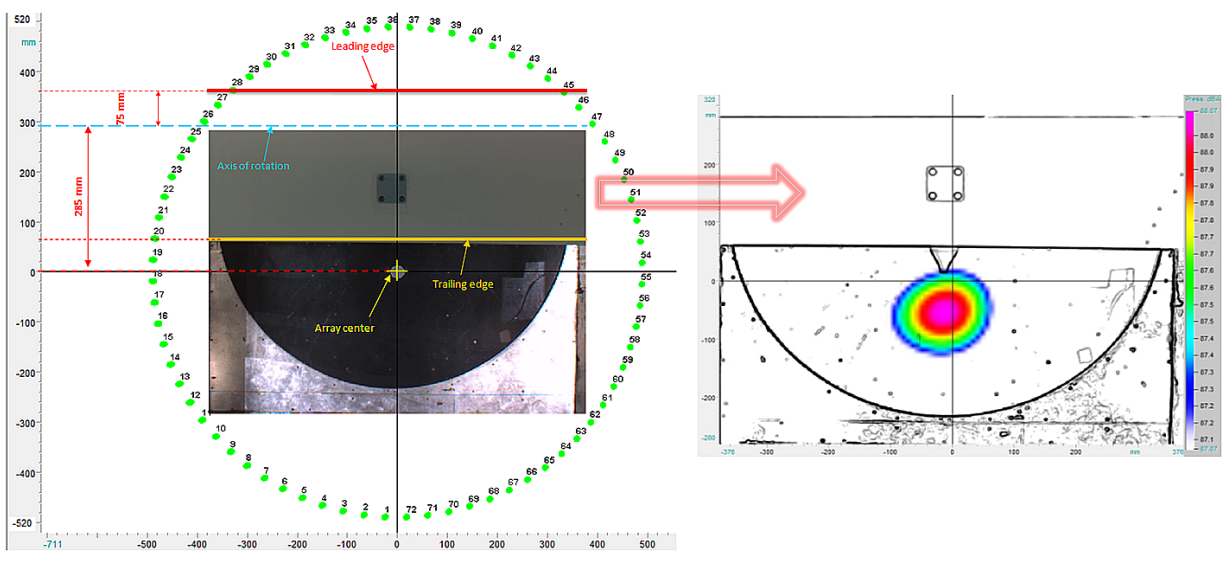

Fig. 8 Wing localization in the WT; noise source localization (integrated over a given bandwidth).

After aeroacoustic tests were completed, and the model was mounted on the external balance supports in clean configuration (with no propulsion units installed), the tests were focused only on aerodynamic loads and pressure 
measurements. The data from the balance are processed according to internal procedures starting from the recorded values.

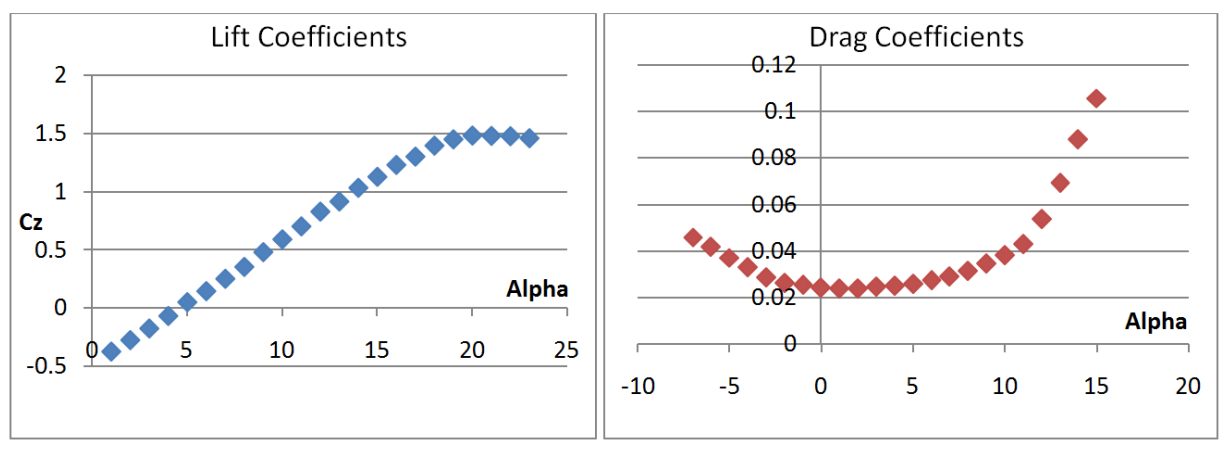

Fig. 9 Lift and drag coefficients

The measured data are corrected with tare (model weight) by subtracting the tare values from the raw data. The measured data has a small influence due to the electrical cables and tubes that are going out from the model on its lateral sides.

\section{B. Aerodynamic and acoustic predictions}

The results presented herein focus on isolated propeller and propeller-wing aerodynamic performance, which serve as an input for noise propagation. Then we focus on the comparison between acoustic measurements and numerical predictions on three cases selected from the global experimental test matrix. The first test selected is the wing alone (clean wing), then we consider the tractor configuration, and finally the pusher configuration. For the two latter cases, only one propeller is present and the sense of rotation is set to counterclockwise (cabin reference). The rotation speed and free-stream velocity are the ones indicated in Table 1.

As introduced above, only the tonal noise related to thickness and steady loading, and the broadband trailing edge noise from the wing and propeller are considered in this study. The contributions of wake and potential interactions will be the subject of future work.

\section{Isolated propeller aerodynamic performance}

The aerodynamic loads on the propeller blades have been obtained according to the numerical method described in Sec. IV.A with the code PUMA. In order to provide a reference to validate the lifting-line results, a Navier-Stokes computation using the Numeca Fine/Turbo CFD package [53] has been performed for the isolated propeller. The steady solver using the $k-\omega \mathrm{SST}$ turbulence model and a $2^{\text {nd }}$ order central scheme has been used. Characteristic boundary conditions have been imposed at the inflow, outflow and far-field faces, while a no-slip condition has been enforced on the blade in the rotating frame. Periodic boundary conditions have been prescribed at the 120 degrees boundaries. To account for low-speed velocities the Merkle low-speed preconditioning has been used.

Figure 10 (left) represents a sector grid of the 13x8-in Master Airscrew propeller. The 20-blocks grid was generated using the Numeca AutoGrid5 grid generator and features 15, 794, 176 cells (fine). The minimum wall distance off the blade surface was $10^{-6} \mathrm{~m}$ and was constant along the whole blade span. The farfield boundaries were positioned at 10 radii $(R)$ from the rotor centre at the inflow and in the lateral farfield boundary, and at $20 R$ from the rotor centre at the outflow. A coarse grid was used for the calculations below and was obtained from fine grid, by removing every second line in all three directions.

As the yaw angle considered in all simulations was 0 degree, all three blades experienced the same inflow conditions regardless of the azimuthal position of the rotor. Therefore, only one blade could be modelled using steady periodicity boundary condition. Figure 10 (right) shows that maximum $y^{+}$was about 1 , which shows suitability of the grid for $k-\omega$ SST turbulence model.

The comparison between the results obtained with both approaches is presented in Fig. 11. Both approaches yield values of the propeller thrust, torque and power in very close agreement. Non-dimensional values of the power and thrust coefficients only differ by about $6.2 \times 10^{-3}$ at most. 

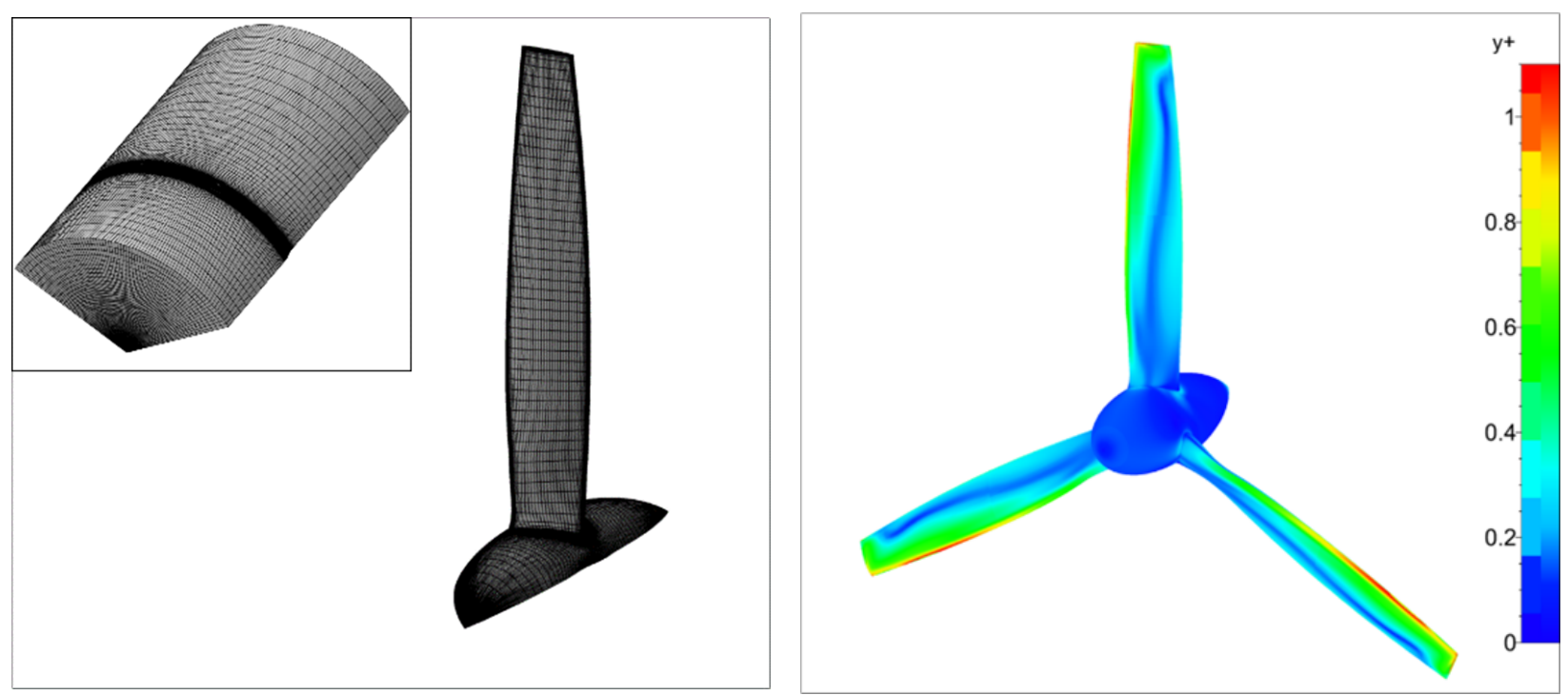

Fig. 10 13x8-in Master Airscrew propeller. (Left) sector grid representation, (right) an example of $y^{+}$plot of the whole rotor.

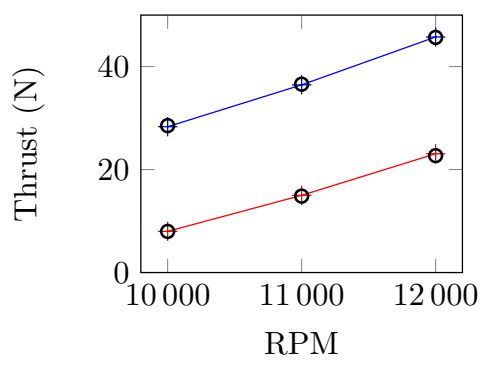

(a) Thrust

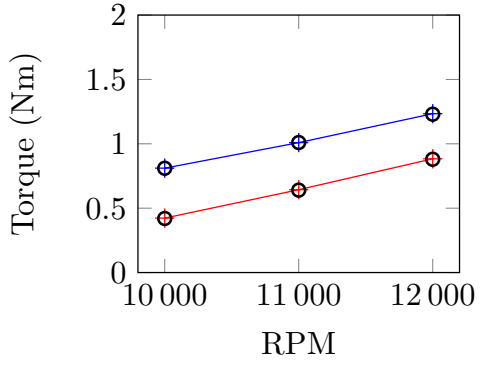

(b) Torque

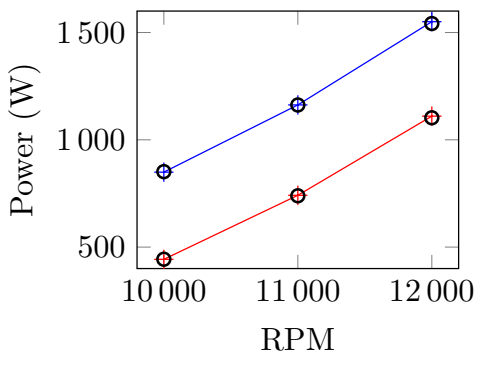

(c) Power

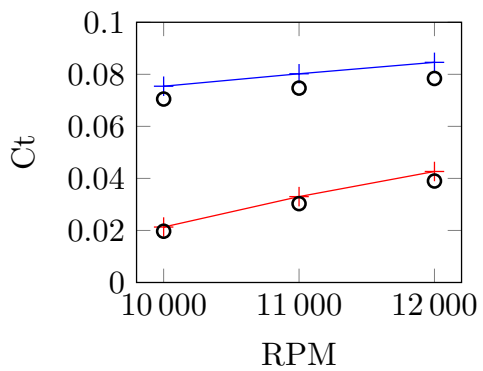

(d) Thrust coefficient

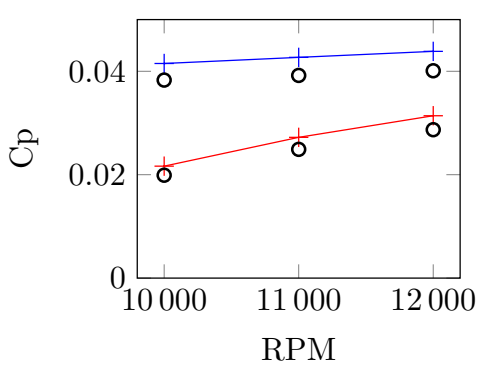

(e) Power coefficient

Fig. 11 Isolated propeller aerodynamic performance at (-) $U_{\infty}=15 \mathbf{m} / \mathbf{s}$ and (-) $U_{\infty}=30 \mathbf{m} / \mathbf{s}$. $\circ$ : CFD, solid lines: PUMA.

\section{Propeller-wing aerodynamic performance}

Modeling propeller-wing interaction requires careful selection of an accurate and fast solution method to capture main unsteady interaction effects. The most conventional choice would be to use the time-domain (TD) Navier-Stokes simulation. However, TD Navier-Stokes simulation of periodic flows requires long runtimes, as several rotor revolutions need to be computed before achieving periodic state. These runtimes can be substantially reduced by solving the governing equations in the frequency-domain (FD). FINE/Open solver of Numeca offers one of the FD methods called the Non-Linear Harmonic (NLH) method, which can model flow unsteadiness at significantly reduced computational 
cost and at comparable accuracy to TD method [54].
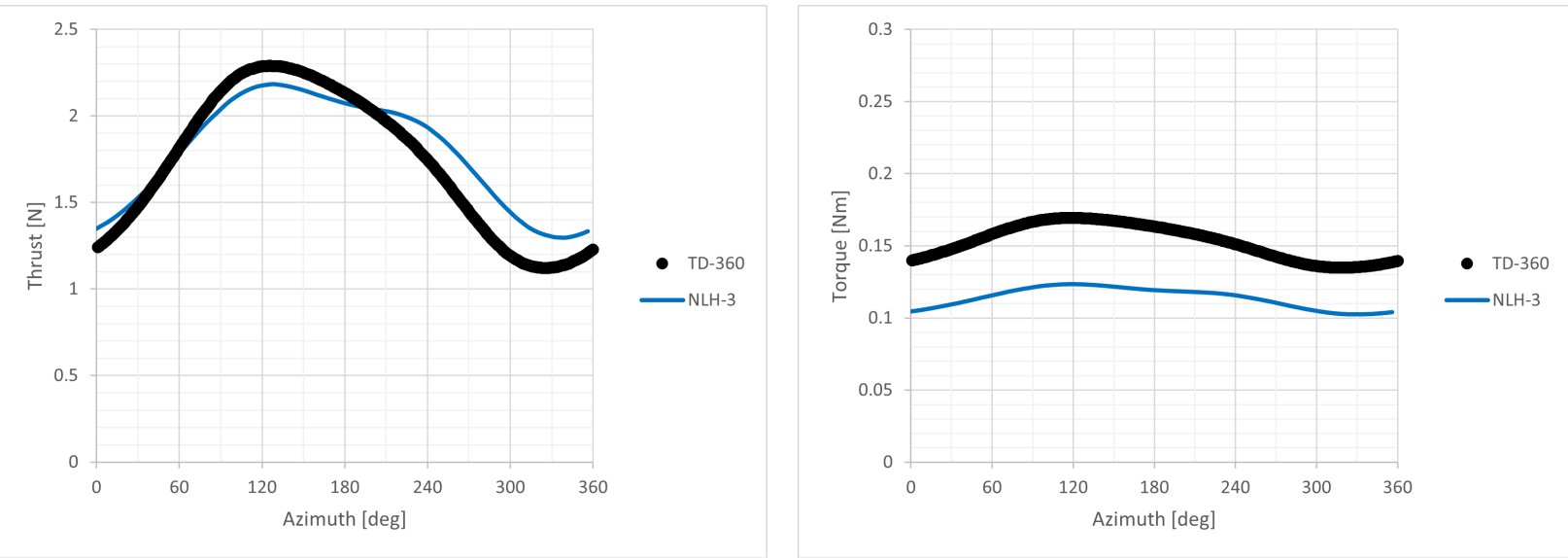

Fig. 12 Evolution of thrust and torque for single blade over one revolution.

Here, the wing and propeller interactions are analyzed with TD simulations using 360 time intervals per period and with the NLH solver using 3 complex harmonics. The periodic profiles of the blade thrust and torque are reported in Fig. 12 One may notice that NLH profiles only slightly differ from the TD-360 profiles. Figures 13 and 14 compare the pressure distribution on the propeller blade surface for the TD and NLH methods when the blade is aligned with the wing leading edge. For pressure distribution only negligible differences can be noticed on both sides of the blade.

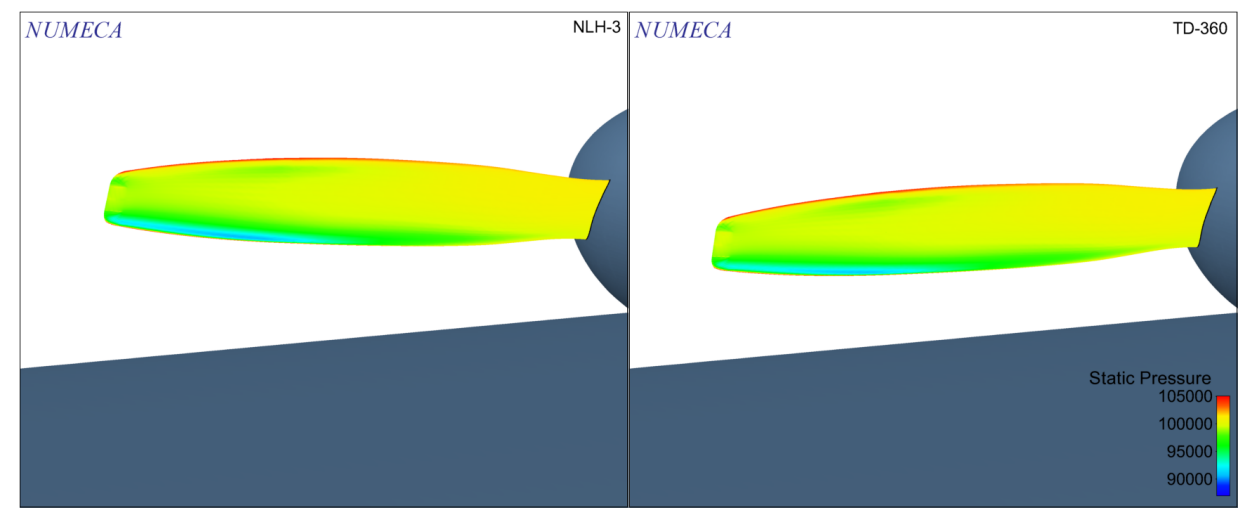

Fig. 13 Static pressure distribution on the pressure side of blade surface (left: NLH-3, right: TD-360).

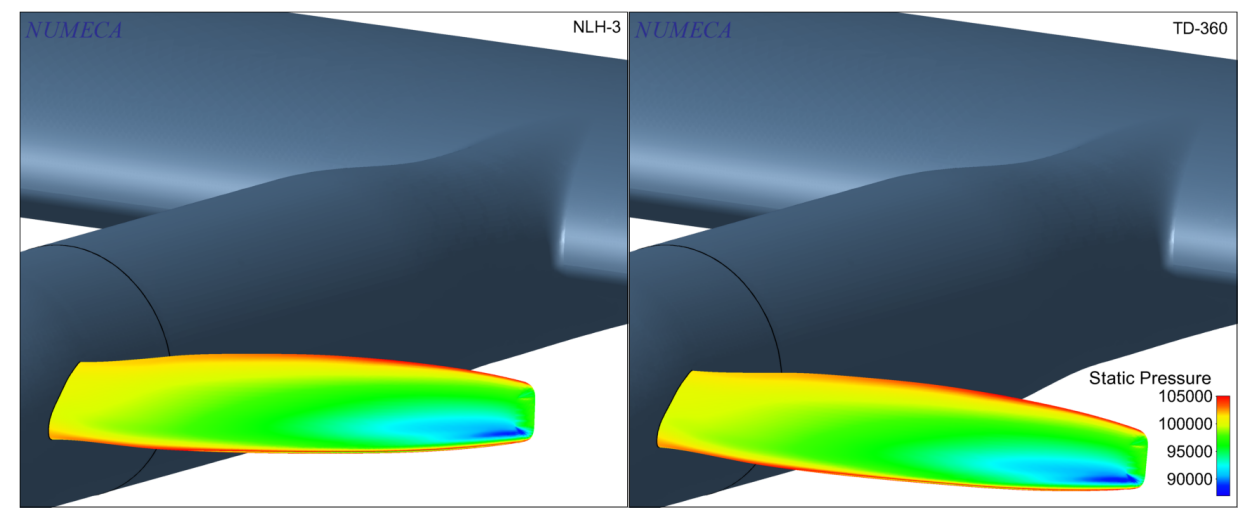

Fig. 14 Static pressure distribution on the suction side of blade surface (left: NLH-3, right: TD-360). 
From an engineering viewpoint, the NLH method can adequately capture the same complex unsteady flow phenomena as the TD method, offering reduction of run time of factor 10. It is expected that three complex harmonics are sufficient to provide a sufficiently converged pressure distribution as an input for noise propagation.

\section{Comparison between numerical predictions and measurements for tonal noise}

The presence of the wing is expected to significantly alter the radiation pattern of the noise emitted by the propeller. This effect should be particularly important when the propeller is in pusher configuration, where specular reflection on the wing suction side and trailing-edge diffraction are expected to occur [55]. On top of these mechanisms, multiple reflections are expected to occur within the wind tunnel test-section as the latter is not anechoic. To fully account for these mechanisms, the acoustic mesh designed for the BEM computation, directly created from the wind tunnel CAD file, had to conform to certain constraints. The first one is that the geometry of the scattering surfaces must be a bounded domain (ie a closed control surface) to be compatible with the Brakhage-Werner integral formulation. This has been achieved by artificially joining the inlet and outlet of the wind tunnel. Secondly, a minimum of 6 elements per acoustic wavelength is needed for the surface discretisation, therefore the test section has been discretised with triangles which edges are about $2 \mathrm{~cm}$ in length, leading to a resolution sufficient for frequencies up to the $4^{\text {th }}$ harmonic of the BPF. The rest of the acoustic mesh is highly stretched in all directions. This allows keeping the total cell count to a manageable value (about 170,000 elements), while ensuring that the results are mostly influenced by the reflections inside the test section rather than possible unwanted edge effects. An illustration of the acoustic mesh used is presented in Fig. 15 where one can notice that most of the grid nodes are concentrated in the portion of the wind tunnel subject to the direct propeller radiation (propeller plane).

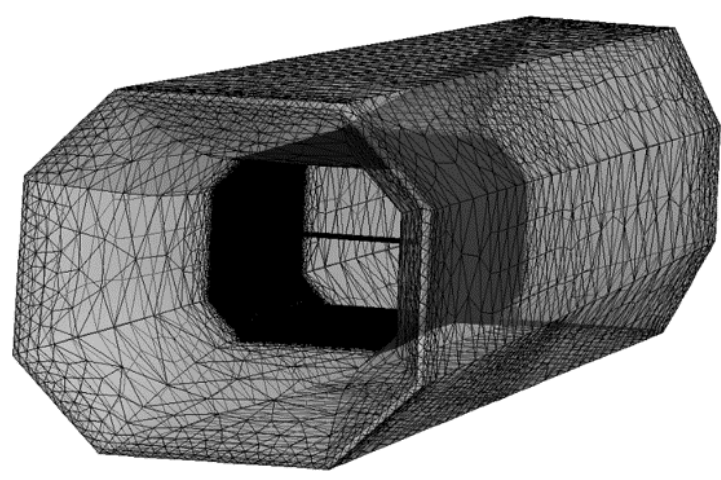

Fig. 15 Acoustic mesh used for the BEM step

The noise levels obtained for the propeller in tractor configuration are presented in Fig. 16 for two opposite microphone positions on the circular array. Please note that, as no microphone is directly on the $x$ axis, the upstream and downstream microphones selected are at an angle of 2.5 degrees from the flow direction.

First, the examination of the "clean wing" measurement allows to see that some tones, possibly due to wind tunnel effects, are present at frequencies slightly higher than the BPF, even when the propeller is not mounted. Nevertheless, apart from the downstream microphone, their amplitude is quite negligible with respect to the one measured at the BPF. To assess the effect of the wind-tunnel walls and wing presence, the SPL values predicted as if the propeller were in free-field (the so-called "direct" field) has also been represented. At the upstream microphone, which is located close to the propeller plane, the free-field predictions are in surprisingly close agreement with the measurements. At the downstream microphone on the other hand, the free-field results are very largely underestimated with respect to the measured amplitude, apart from the BPF. As for the BEM results, we observe some discrepancies with respect to the measurements, with a large overestimation for the first two harmonics, and a smaller underestimation of the $3^{\text {rd }}$ and $4^{\text {th }}$ harmonics at the upstream microphone. At the downstream microphone, quite similar observations can be made. Despite some differences with measured tones amplitudes, the BEM results seem to globally improve the agreement with the measurements.

In addition to these single microphone comparisons, directivity plots are presented in Fig. 17, representing the comparison between measurements and numerical estimations of the tones levels on the circular array. The angular convention is such that 0 degrees corresponds to the downstream microphone. In spite of the discrepancies observed on 


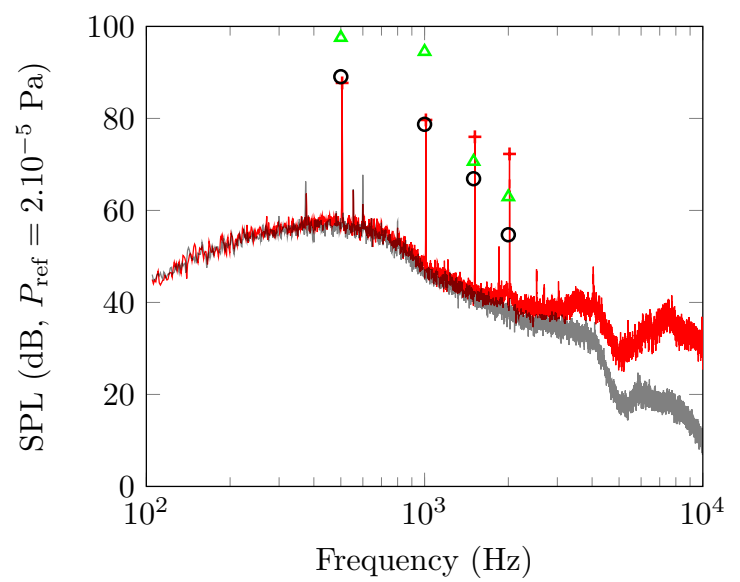

(a) Upstream microphone

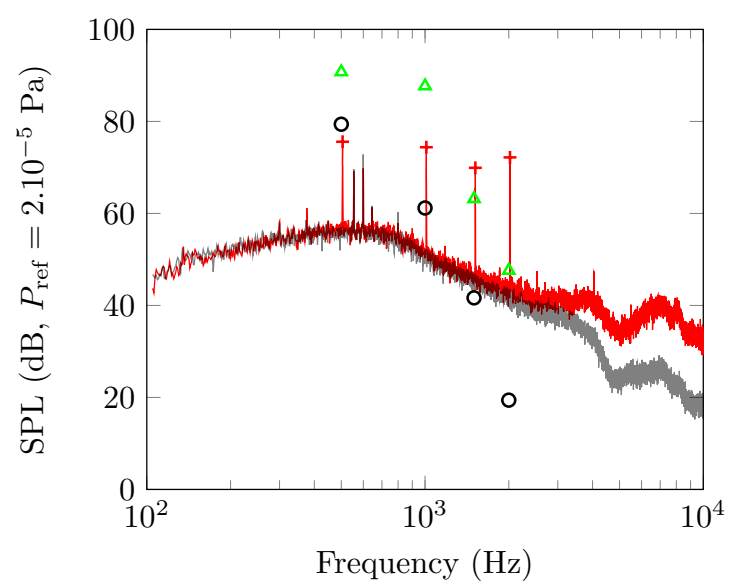

(b) Downstream microphone

Fig. 16 Tractor configuration, $R P M=10000, U_{\infty}=30 \mathrm{~m} / \mathrm{s}$ - comparison between the experimental (-) and numerical noise levels. $\circ$ : propeller in free-field, $\triangle$ : propeller with the wing and wind tunnel walls. $(-)$ : clean wing.

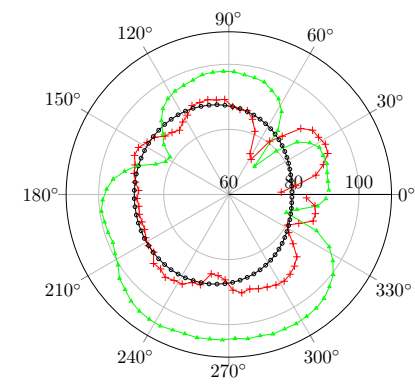

(a) BPF1

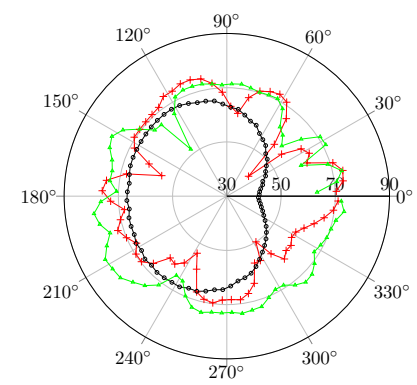

(c) BPF3

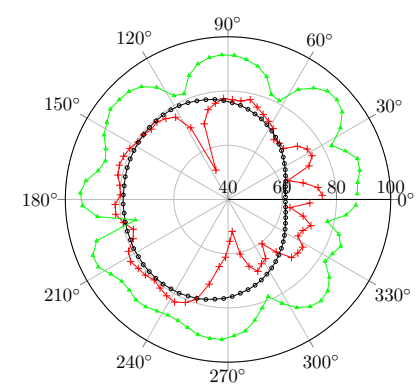

(b) BPF2

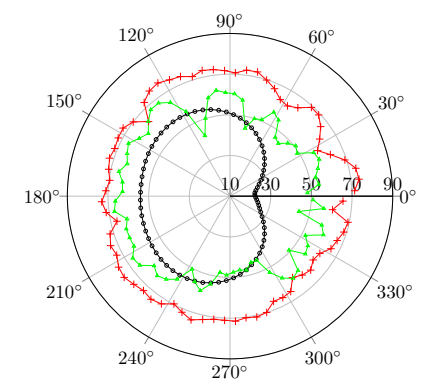

(d) BPF4

Fig. 17 Tractor propeller. Comparison between measured and predicted noise levels at the BPF harmonics on the circular microphone array. $\circ:$ propeller in free-field, $\Delta:$ propeller with the wing and wind tunnel walls, + : measurements.

the isolated microphones, the computed directivity shape seems to present some common points with the measured one, as both datasets present some common directions of relative extinction, such as for instance BPF1 at about 60 degrees, or BPF3 at about 45 and 240 degrees.

On the pusher configuration results, shown in Fig. 18, similar observations can be made. Please recall that this time, the microphone array is in the shadow region of the propeller and therefore installation effects are expected to play an 


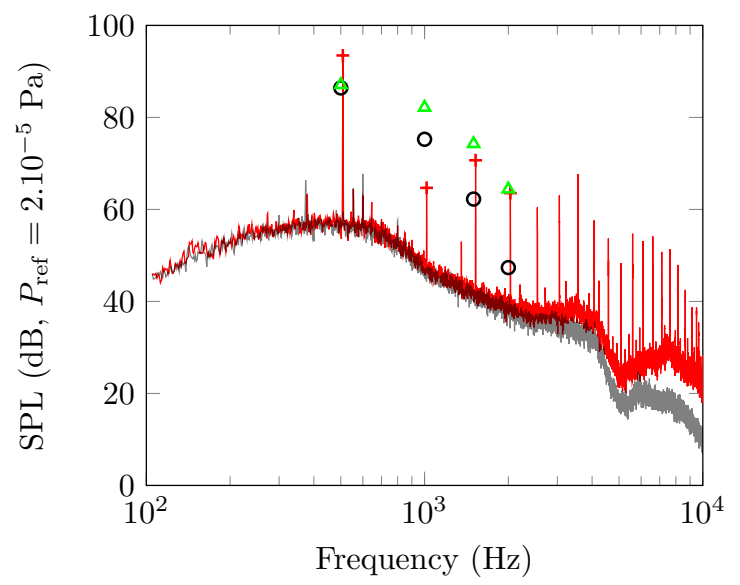

(a) Upstream microphone

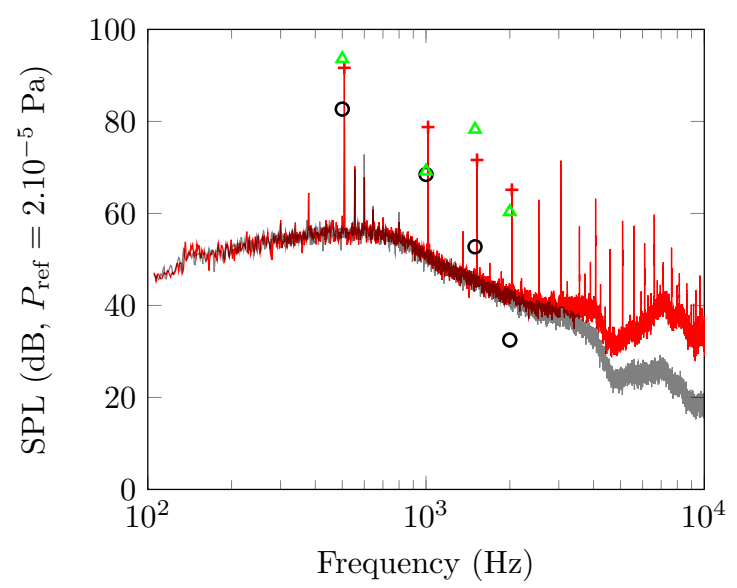

(b) Downstream microphone

Fig. 18 Pusher configuration, RPM=10000, $U_{\infty}=30 \mathrm{~m} / \mathrm{s}$ - comparison between the experimental (-) and numerical noise levels. $\circ$ : propeller in free-field, $\triangle$ : propeller with the wing and wind tunnel walls. (-): clean wing.

important role. Another difference with the tractor configuration is the impingement of the pylon wake on the propeller blades that seems to significantly enhance high order harmonics, as observed experimentally by Sinnige et al [56] on a similar pylon-propeller configuration. While the free-field results again globally underestimate the tones levels, the discrepancies between experiments and BEM results seems less severe. Recalling that the pylon effect is not accounted for in the present numerical methodology, it is possible that some of the remaining differences could be due to this unsteady mechanism.

Regarding the directivity on the circular array, shown in Fig. 19, we can once again observe angular regions of relatively good agreement between measured and computed directivity shapes, especially at the $4^{\text {th }}$ harmonic, although large over-estimations of the first harmonic amplitude are still observed for microphones located under the wing.

To summarize, tonal noise predictions have been globally improved by taking into account the wind tunnel walls and the wing surface by using a BEM computation. The agreement with measurements is more favorable for the pusher configuration, while some discrepancies remain for the tractor configuration. These discrepancies have been investigated by verifying the ability of the method to correctly propagate a simple source inside a confined environment (see appendix VI. A possible source of error is that in the BEM computations, all the walls are considered as perfectly rigid, which is not the case everywhere in the test section.

\section{Comparison between numerical predictions and measurements for broadband noise}

As described in Sec. IV] the broadband trailing-edge noise methodology relies on RANS computations performed at predefined iso-radii according to the adopted strip approach, as illustrated in Fig. 20. Rotor flow dynamics usually have a highly three-dimensional behaviour, typically due to unbalance of centrifugal and pressure forces. However, in our case, since the propeller blades are almost straight, without lean or sweep, the flow is expected to be mostly two-dimensional at a specific iso-radius justifying the use of 2D computations.

The two-dimensional flow computations are performed using StarCCM+13.04 with the $k-\Omega$ turbulence model. Only one blade is considered in the flow domain, with periodic boundary conditions on top and bottom boundaries, as illustrated in Fig. 20. The velocity inlet condition is imposed as the free-stream wind tunnel velocity in the streamwise direction $(30 \mathrm{~m} / \mathrm{s}$ in the present case) and the rotational speed velocity in the cross-wise direction. Zero-pressure condition is used at the domain outlet. Using those boundary conditions, installation effects due to azimuthal flow non-uniformities cannot be taken into account in the present methodology while this might have an effect on the noise predictions, especially for the pusher configuration where the influence of the wing on the flow deviation is increased. The polyhedral mesh is created on a domain extending from $5 C$ upstream and $20 C$ downstream of the propeller trailing-edge, for use of wake information for wake interaction noise in future work. The mesh is refined at the propeller wall to reach $y+<1$ with 20 prism layers and a refinement bloc is added, aligned with the wake, to attempt to 


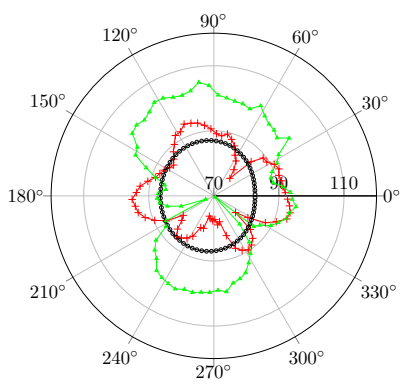

(a) BPF1

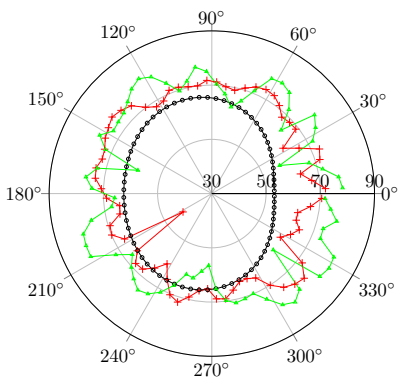

(c) $\mathrm{BPF} 3$

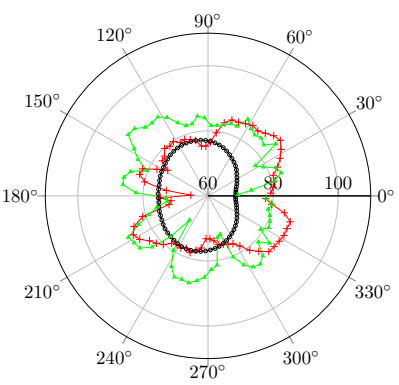

(b) BPF2

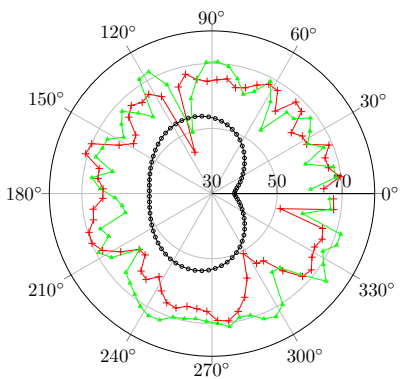

(d) BPF4

Fig. 19 Pusher propeller. Comparison between measured and predicted noise levels at the BPF harmonics on the circular microphone array. $\circ$ : propeller in free-field, $\Delta$ : propeller with the wing and wind tunnel walls, + : measurements.

minimize numerical diffusion errors in the wake evolution. As an example, for the mid-span location mesh for which the velocity magnitude iso-contours are shown in Fig. 20 (right), the number of cells are about $0.9 \mathrm{M}$.

As a first step, the pressure coefficient distribution between the three dimensional NLH computation is compared with the results obtained by two dimensional iso-radius RANS computations in Fig. 21. The overall behavior of the pressure evolution along the chord is reproduced for both mid-span and tip locations. The main differences, especially in terms of pressure gradient, between both numerical methods are appearing in the trailing-edge region where the inputs for the broadband noise trailing-edge method are extracted. The influence of the wall-pressure gradient on the wall-pressure spectrum has been largely investigated in literature [36] but a deeper investigation of the sensitivity of the noise results should be addressed in future work.

The results shown in Section V.B.3 have shown the importance of accounting for the wind tunnel reflections when simulating the tonal noise emissions. The same exercise has been carried out for the broadband noise radiated by the wing (without propulsor), using now the Simcenter 3D Acoustics FEM solver to calculate the ATVs along the procedure depicted in Section IV.B. As for the tonal wind tunnel correction that was performed using the BEM solver, it is assumed that the acoustic field radiated towards the inlet and outlet of the straight wind tunnel branch containing the test component is not re-injected through the other end. A first computational domain has thus been considered, containing only this straight wind tunnel branch, convergent and diffuser included, and both terminated by non-reflective Automatic Matched Layer boundary conditions. The volume is meshed using hexahedral elements with $0.05 \mathrm{~m}$ cell size, leading to a numerical cut-off frequency of $1,133 \mathrm{~Hz}$ as per the six-elements-per-wavelength criterion. A coarser mesh could have been used for a direct frequency response analysis, exploiting the ability of the FEM Adaptive Order solver, but the ATVs being exported on the surface mesh, they would still have to respect the same six-elements-per-wavelength criterion. The standard Nastran FEM solver was therefore employed. This FEM mesh, shown in Fig. 22 (top-right), contains about 1.4 million cells. To conclude the FEM analysis of the wind tunnel reflections, an additional numerical test has been conducted in an attempt to reach higher analysis frequencies, with a finer FEM mesh including only the part of the test section containing the microphone antenna. With hexahedral elements of $0.018 \mathrm{~m}$ size, this mesh of 1.4 million elements as well can reach $3,150 \mathrm{~Hz}$ (Fig. 22, bottom-right).

The results shown in Fig. 22 indicate that if the levels predicted for the wing alone radiation assuming free-field 

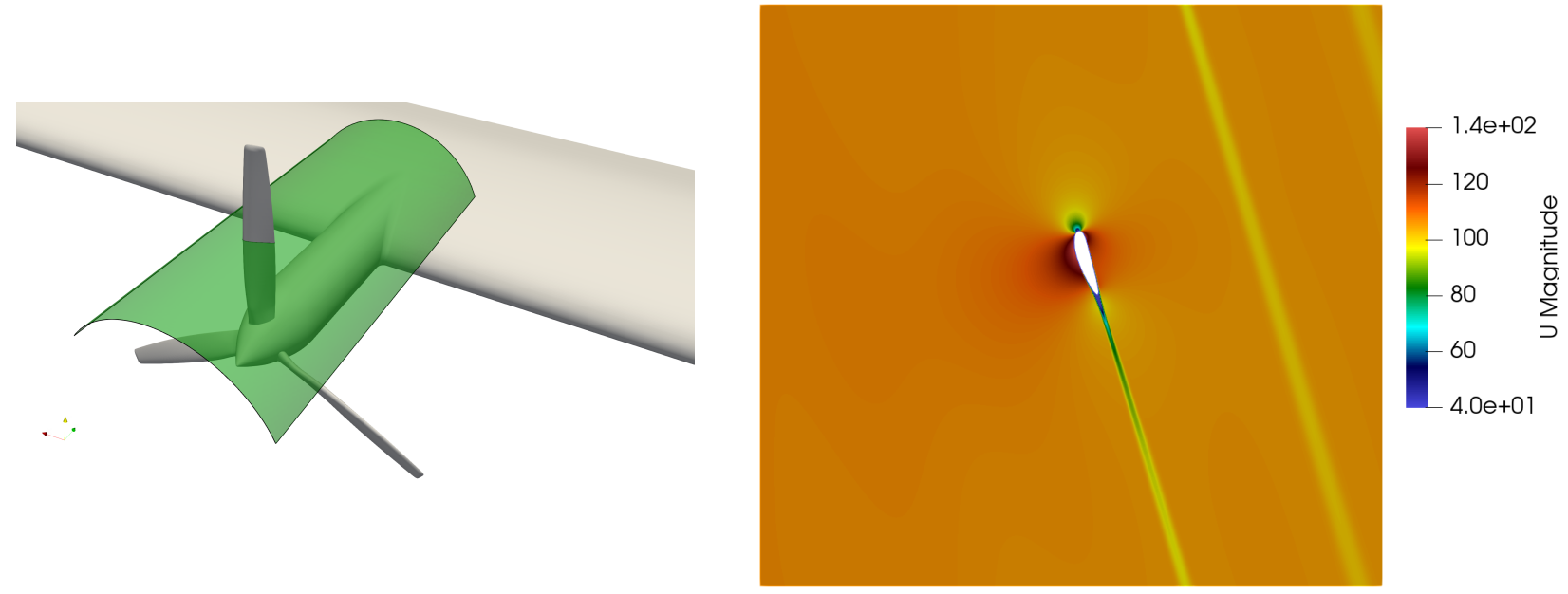

Fig. 20 (left) Iso-radius cut of the propeller blade, defining the computational domain and (right) resulting computed flow around the blade and downstream of the propeller.
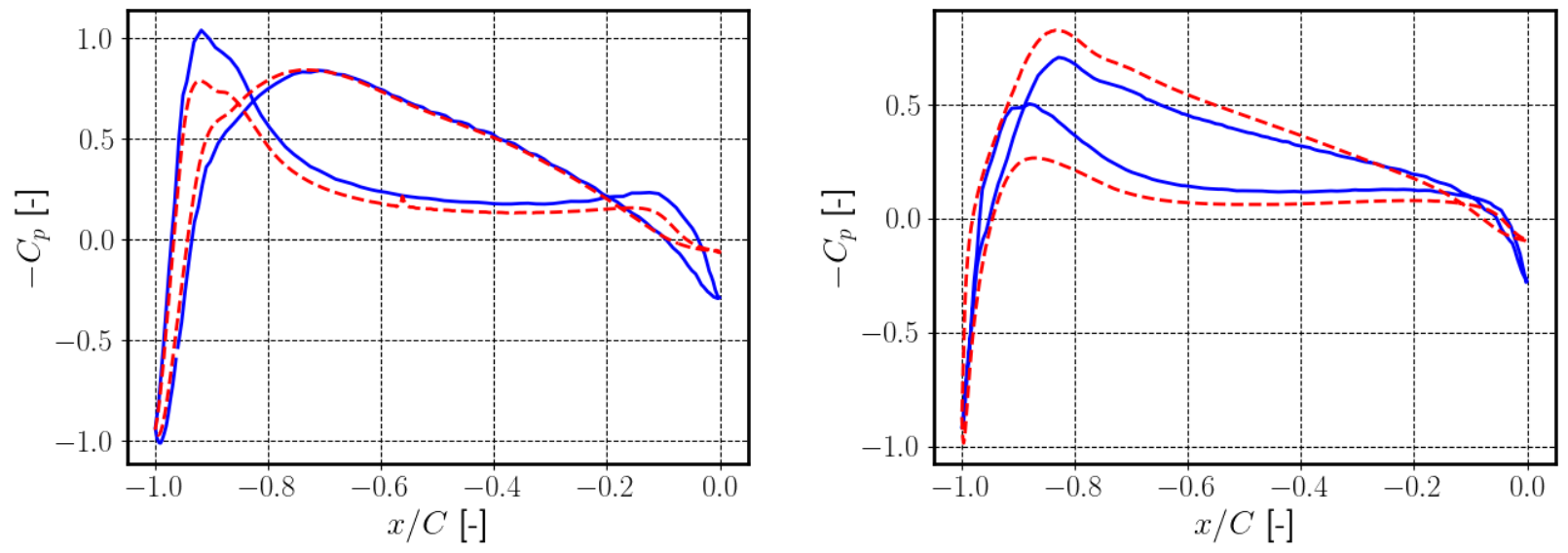

Fig. 21 Comparison of pressure coefficient distribution between 3D NLH computations (dashed) and 2D iso-radius RANS (plain) computations at (left) mid span and (right) tip location. 

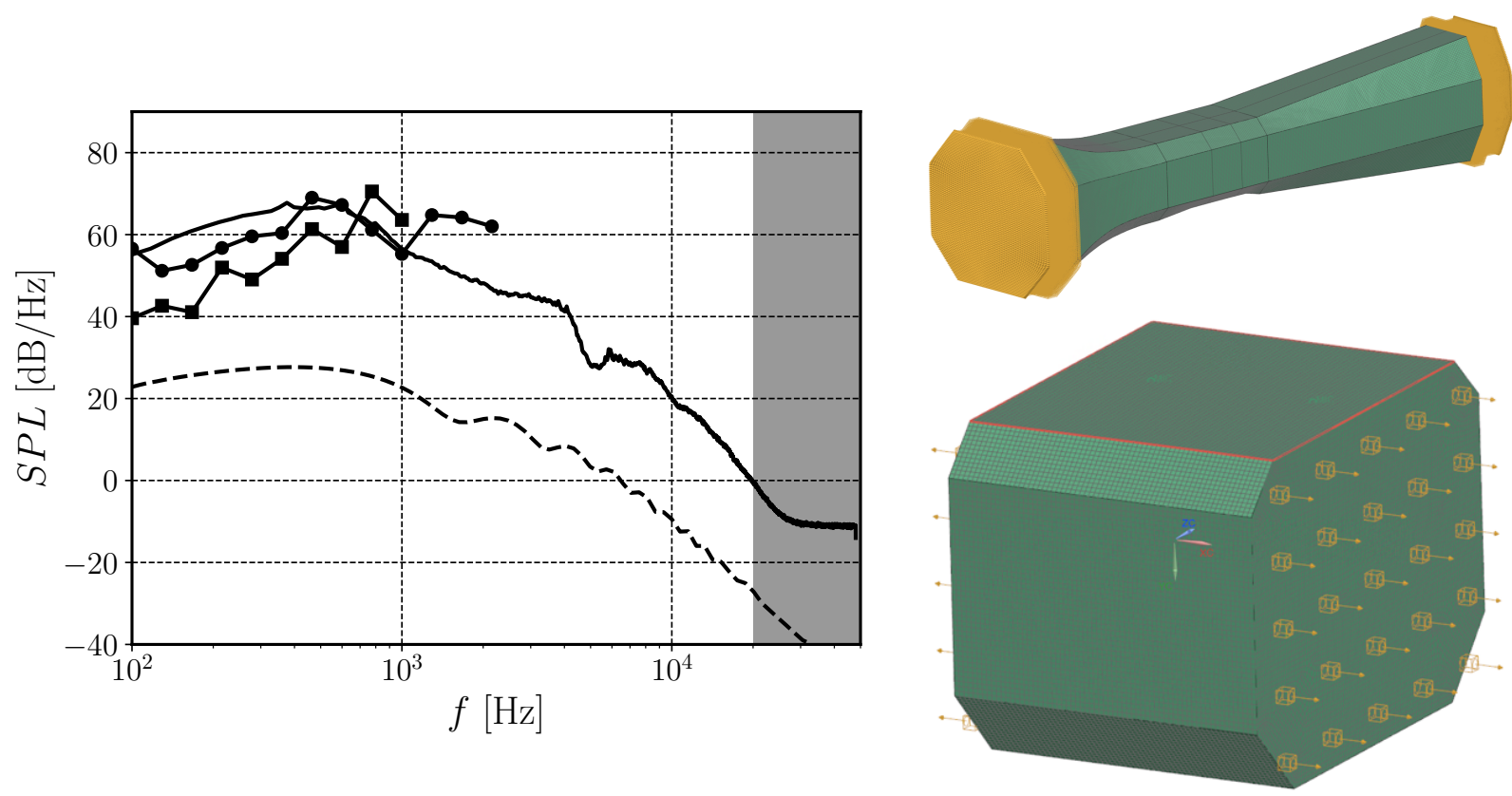

Fig. 22 (left) Sound pressure level of the sound radiated at the upstream observer for the wing alone experiment (plain), the free field wing prediction (dashed), the wing predictions taking wind tunnel effects into account using the complete wind tunnel FEM model with $1,133 \mathrm{~Hz}$ maximum frequency (square) and using the reduced wind tunnel FEM model with (circle).

conditions were largely under-estimating the measured ones, accounting for the wind tunnel reflections brings a much improved agreement. The discrepancies between the numerical results obtained on the two different meshes require further analysis, but aren't completely unexpected considering the differences of surface contributing to the reflections. In any case, accounting for the wind tunnel reflections at larger frequencies will require a tailored modelling strategy, combining the efficiency of the FEM-AO solver with the ATV mesh requirements at the wall. Furthermore, it should be also noted that the wind tunnel walls are considered as hard-walls and then perfectly reflecting, while this is obviously not the case in the experiments where the walls are partly treated with acoustic absorbent material.

The same Amiet-ATV methodology was applied for the analysis of the acoustic reflections of the propeller TE noise by the wing. In this instance the wind tunnel reflections aren't accounted for. In order to model the propeller noise source, 5 strips have been used and the same methodology as described in Fig. 6 has been applied. For every strip, a RANS computation has been run using the same parameters as above, and the Lee model [36] was used to compute the wall-pressure spectrum injected in Amiet's theory in rotation [42]. The FEM mesh is shown in Fig. 23. and contains about 500,000 tetrahedral elements. An AML boundary condition has again been prescribed at the outer boundary of the mesh, but this time a Kirchhoff radiation condition has been applied to this outer surface since the listener points are located outside of the FEM domain.

The results of these last simulations are compared with the measurements in Fig. 24 for two microphone locations within the antenna, on a line that is $0.021 \mathrm{~m}$ off the wind tunnel vertical symmetry plane, upstream and downstream of the wing TE. The frequency band where the microphones experienced measurement frequency cut-off is highlighted in grey. It should be first noted that the predicted levels are much below those measured in the wind tunnel, by approximately the same amount as for the wing alone. It is safe to assume that the difference is again due to wind tunnel reflections. The analysis will concentrate on the relative contribution of the propulsor, when added to the wing intrinsic source. The measurements highlight tonal and broadband contributions of the propeller, on top of the spectrum of the wing alone. The tonal contribution of the tractor configuration appears at the BPF and its first three harmonics, while the pusher propeller introduces a much richer tonal content extending nearly up to $10 \mathrm{kHz}$. It can be conjectured that the pylon wake is introducing a distortion of the flow ingested by the propeller with fairly high azimuthal orders. In addition to the tonal noise, the propeller contributes also to the radiated broadband noise and dominates the wing radiated noise above about $2 \mathrm{kHz}$ for all configurations and microphone positions as shown in Fig. 24 

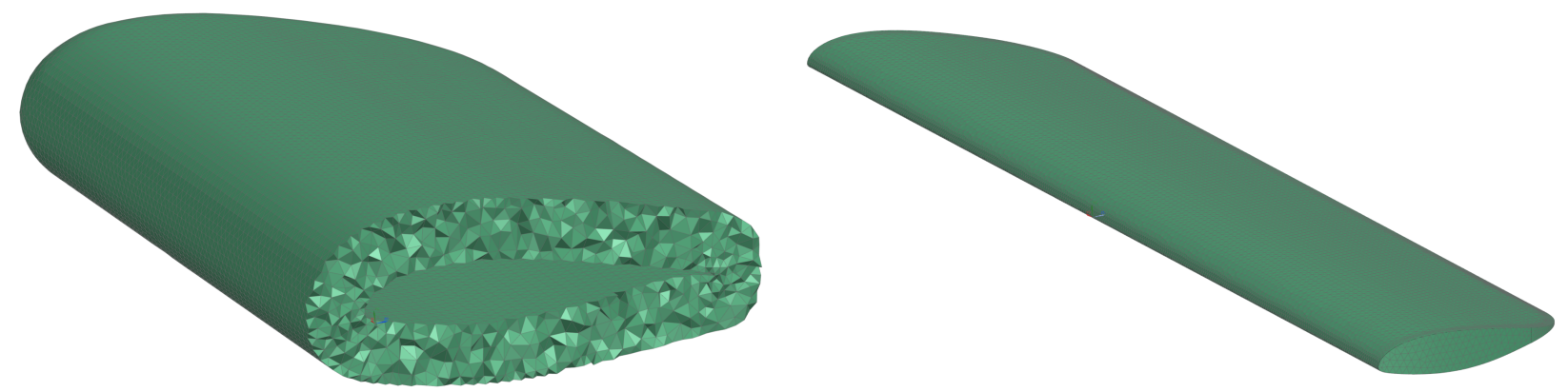

Fig. 23 (left) Mid-span cut of the volumetric FEM model of the main wing and (right) the corresponding wall-surface mesh.

The numerical predictions show a fair agreement compared to the experiments in terms of relative SPL between the wing and propeller configuration and the wing alone experiments. Similarly to the experiments, the propeller broadband noise starts to dominate the wing noise for frequencies above $2 \mathrm{kHz}$. This is mainly due to the high rotational speed of the propellers, for which the application of the traditional wall pressure models remains questionable given the high adverse pressure gradient observed near the trailing edge (as shown in Fig:21) and the small local flow recirculation present in that area. The faster decay of the propeller noise at higher frequencies, observed in the experiments but not reproduced by the numerical results, might be attributed to that poor modelling of the wall pressure methods in such conditions. This would require further study on dedicated controlled test cases.

The effect of the scattering of the propeller radiated sound on the main wing is also illustrated in Fig. 24, in the frequency range of $1-5 \mathrm{kHz}$. For the tractor configuration, taking into account the main wing increases the total radiated sound up to $4 \mathrm{~dB}$ for both upstream and downstream microphones. This is mainly due to the propeller position relative to the wing that is not supposed to lead to particular shielding effects, resulting in a similar wing effect for both microphones. For the pusher configuration, the propeller is shielded by the wing for the upstream microphone, and results in larger effects on the radiated noise compared to other configurations. A decrease of the noise might be expected but the numerical results show an increase of the noise in the investigated frequency range that would correspond to a noise regeneration due to the main wing scattering for that particular observer position. For the downstream microphone, the effect of the main wing is negligible as observed in Fig. 24(d). This could be explained by the microphone position that is located away downstream from the tested components, making the direct noise coming from the propeller dominant. For the pusher configuration, the presence or absence of observed sound regeneration should be further analysed by computing the complete sound directivity around the investigated wing and propeller configuration.

\section{Conclusions and perspectives}

Distributed Electric Propulsion systems involve a variety of single- and multi-body interactions that can yield beneficial or adverse aeroacoustic effects. A simulation framework is being developed to predict those interactions, the focus being placed in this work on the wing and single propeller self-noise, associated with the trailing-edge, steady loading and thickness noise contributions. The validation data was provided through wind tunnel tests with reflecting walls, for which numerical acoustic models were built to permit meaningful comparisons.

The overall simulation strategy was based on mid-fidelity methods, aimed at providing reasonably accurate predictions at moderate CPU costs, in the perspective of performing parametric studies and optimization in a near future. Tonal noise has been computed with a simulation chain featuring three in-house ONERA codes, respectively devoted to determining the blade loading using a lifting line method, computing the direct field at the listeners and scattering surfaces, and finally computing the total (including scattering/reflections) field. The broadband noise simulations were carried out using VKI in-house tools based on Amiet's semi-analytical models and combined with Acoustic Transfer Vectors obtained from numerical acoustics solutions.

The simulation methods were shown to yield a fair agreement with the wind tunnel acoustic spectra, opening promising perspectives for the cross-validation of aeroacoustic simulations and reverberant wind tunnel noise measurements. The computational efficiency of the numerical acoustic methods remains however challenged by the dimensions of the scattering surfaces and the frequency range of interest (up to several kilohertz), and should be improved through the application of advanced numerical implementations. 
(a)
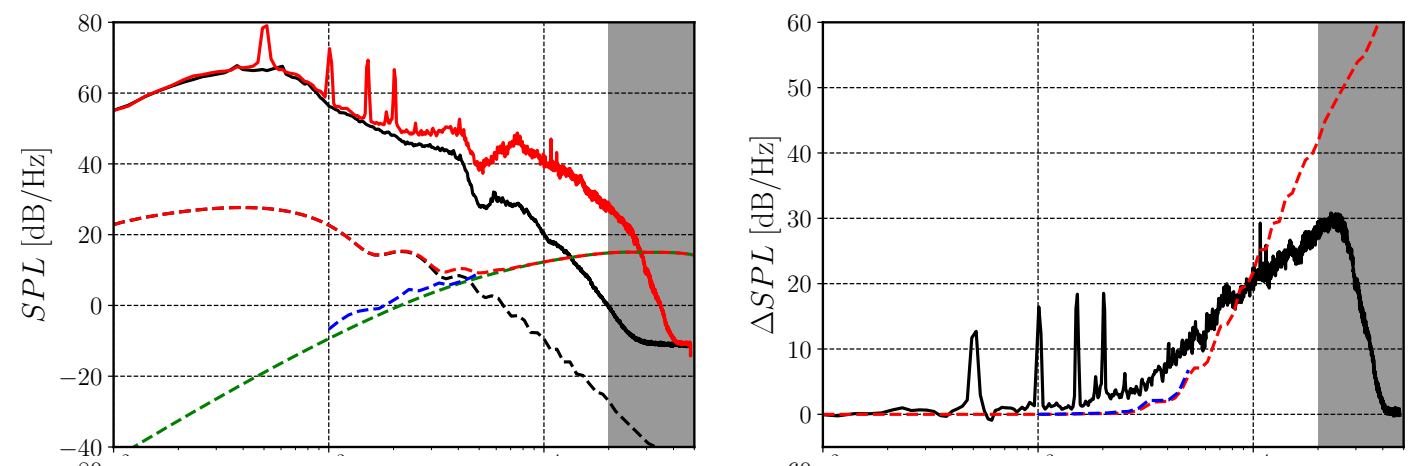

(b)
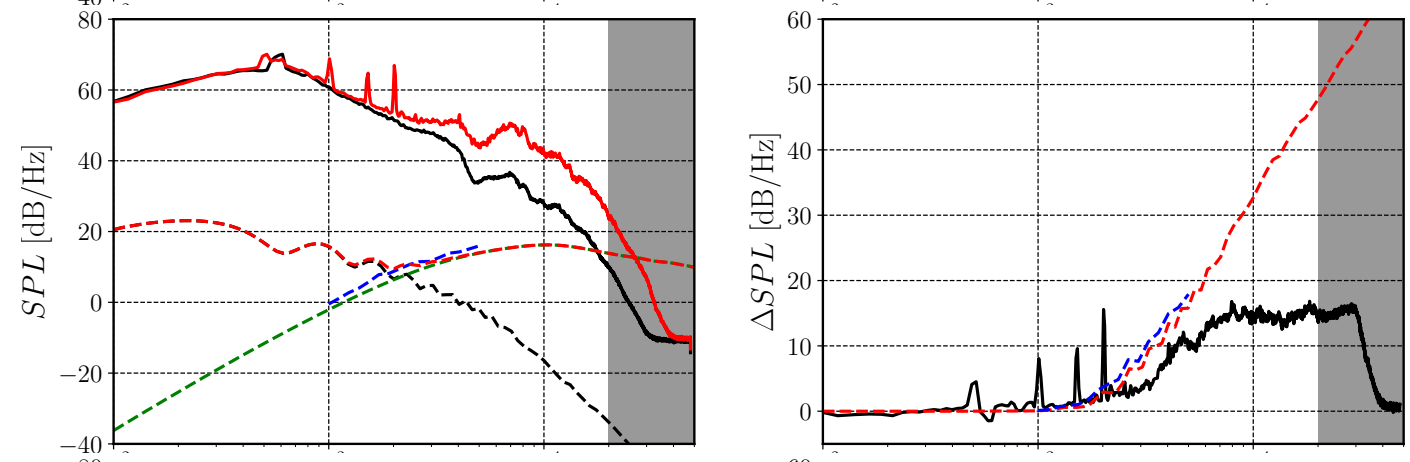

(c)
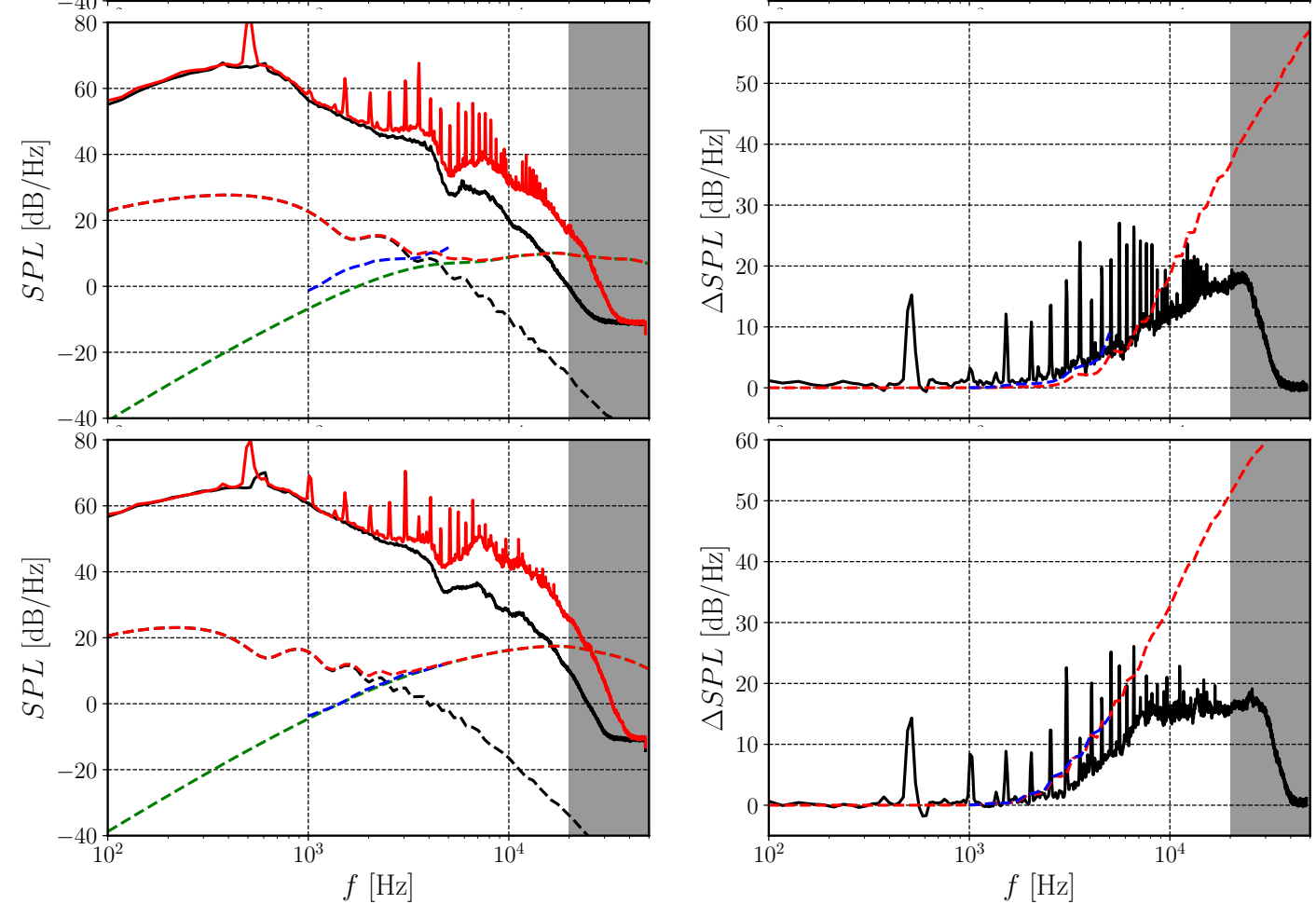

Fig. 24 Comparison of the numerical predictions (dashed) with the experiments (plain) for the tractor configuration at the (a) upstream and (b) downstream microphones, and for pusher configuration at the (c) upstream and (d) downstream microphones. (Left) Spectra of sound pressure level of the wing (black), the propeller (green), the wing and propeller (red) and the propeller and wing scattering effects (blue) configurations. (Right) delta SPL between wing and propeller and wing configurations.

Future work on DEP systems will consider multi-body installation effects that were not addressed in this study: potential interaction and wake interaction noise. The current framework, based on (U)RANS simulations and semi- 
analytical models, should remain suitable for the relatively simple geometries composing the current DEP tractor and puller mock-ups.

\section{Acknowledgments}

A.H. thanks Dr. Luis Bernardos Barreda (ONERA Meudon, DAAA) for providing the airfoil polars and for his assistance with the PUMA computations. The kind support of Dr. Korcan Kucukcoskun for the Simcenter 3D simulations is gratefully acknowledged as well.

This project has received funding from the European Union's Horizon 2020 research and innovation program under grant agreement No 769350.

\section{Appendix: Validation and comparison of BEM and FEM for acoustic propagation inside the wind tunnel}

Whilst acoustic measurements are traditionally performed in anechoic environments, the results presented in this paper have been obtained in a closed test-section wind tunnel. Under these circumstances, making accurate noise predictions is a challenging task that requires taking into account different boundary conditions than the traditional free-field radiation condition.

In the recent years, some research has been conducted at ONERA to be able to properly simulate sound propagation in aerodynamic wind tunnels. One of the outcomes of this research is the numerical strategy based on BEM described and used in this paper. As a complement, VKI has used the FEM solver of the Simcenter 3D simulation platform developed by Siemens [45], featuring Adaptative Order finite elements (FEM-AO, [50, 51]) and Automatic Matched Layers (AML, [52]) for non-reflective boundary conditions. Such conditions are prescribed in the present study based on the assumption that acoustic waves propagating away from the test section are damped out and not re-injected through the closed loop wind tunnel. The prescription of non-reflective boundary conditions in a BEM context is less straightforward, especially when the mesh needs to be closed without free edges as in the ONERA BEM code.

A validation of both solutions is presented below for an elementary test case which consists in a monopole source placed in a straight duct of dimensions comparable to the INCAS wind tunnel. The mesh resolution is the same as that used for the INCAS wind tunnel simulations. The monopole radiation is calculated at frequencies corresponding to the blade-passing frequency harmonics in the experiments. More specifically, the duct considered has a rectangular cross-section of dimensions $a=2.5 \mathrm{~m}$ in the $x$ direction and $b=2 \mathrm{~m}$ in the $y$ direction. The source position is arbitrarily chosen as $\left(x_{0}, y_{0}, z_{0}\right)=(1.9,0.3,1.4) \mathrm{m}$ in the Cartesian frame with origin in the corner of the duct. The complex acoustic pressure is computed on the straight line defined by $(x, y)=(1.25,0.4) \mathrm{m}$ for $-7.5<z<7.5$. This case presents the advantage of having an analytical solution, allowing the assessment of the precision obtained on the numerical result. Indeed, the Green's function tailored to this geometry is given by [57, 58]:

$$
G=\frac{i}{2 a b} \sum_{n=0}^{\infty} \sum_{m=0}^{\infty} \frac{c_{n} c_{m}}{k_{n m}} \cos \left(\alpha_{n} x\right) \cos \left(\beta_{m} y\right) \cos \left(\alpha_{n} x_{0}\right) \cos \left(\beta_{m} y_{0}\right) e^{ \pm i k_{n m}\left(z-z_{0}\right)}
$$

where $c_{n, m}=2$ for $n, m=0$ and 1 otherwise, $\alpha_{n}=n \pi / a, \beta_{m}=m \pi / b$, and $k_{n m}=\sqrt{k^{2}-\alpha_{n}^{2}-\beta_{m}^{2}}$ with $\mathfrak{R}\left(k_{n m}\right) \geq 0$ and $\mathfrak{J}\left(k_{n m}\right) \leq 0$. Finally, considering the low Mach numbers in experiments and for simplicity reasons, the results presented hereinafter are all obtained in the absence of flow.

The comparison between the analytical and numerical solutions is presented for BEM and FEM at two frequencies, $500 \mathrm{~Hz}$ and $1 \mathrm{kHz}$, in Figs. 25 and 26, respectively. Despite some small discrepancies on the BEM solution, both numerical methods return the complex pressure amplitude with very satisfying accuracy. A possible explanation of the better accuracy of FEM in this case is the use of automatic matched layers at the physical ends of the duct, while the mitigation of possible end effects in BEM are handled with a progressive stretching of the acoustic mesh, which might result in some spurious contributions in the total field, especially in the long wavelength limit. 

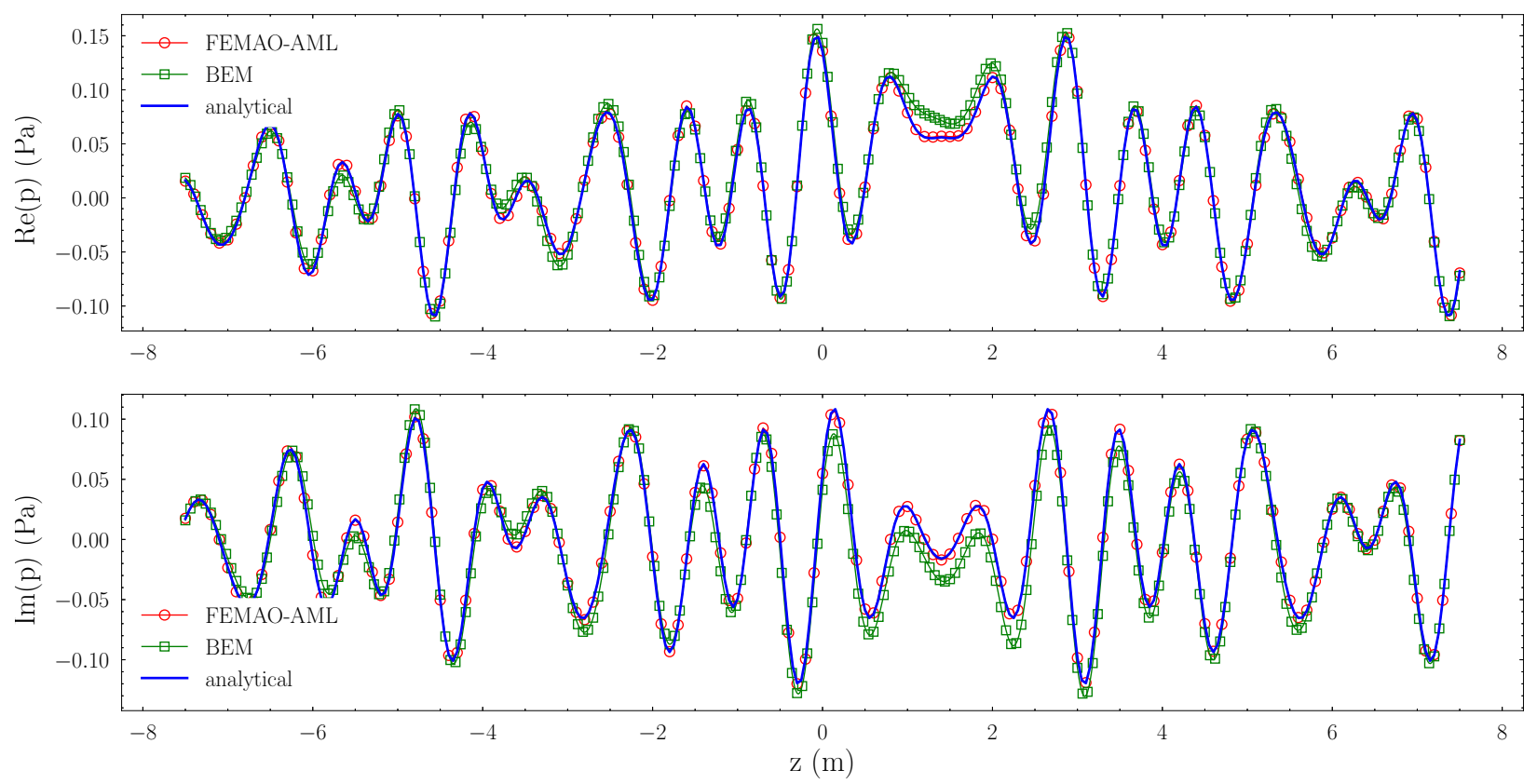

Fig. 25 Complex pressure on the observer line at $500 \mathrm{~Hz}$.
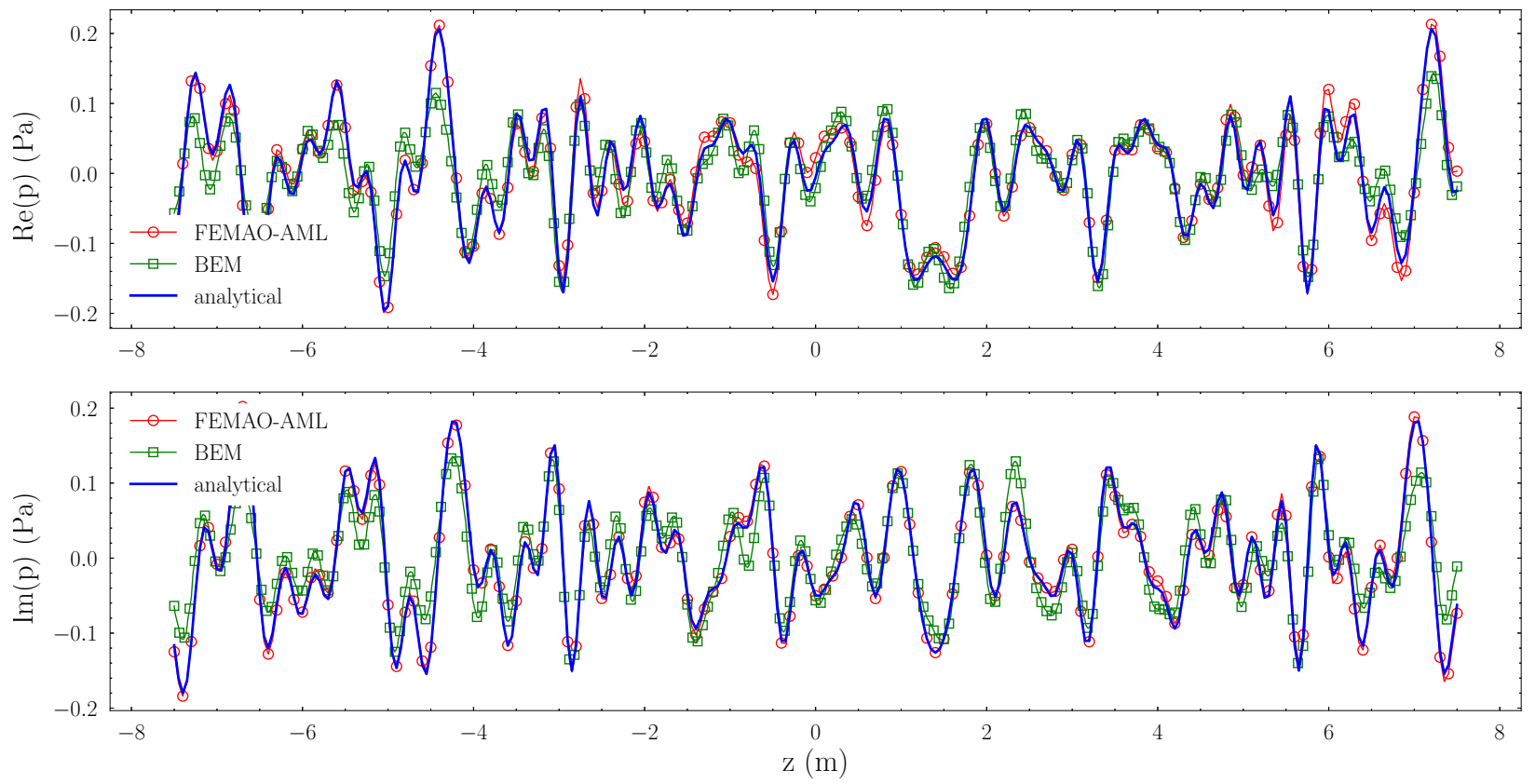

Fig. 26 Complex pressure on the observer line at $1000 \mathrm{~Hz}$.

\section{References}

[1] Civil Aviation Authority, G. A. S., "Emerging Aircraft Technologies and their potential noise impacts,", 2019. URL http: //publicapps. caa. co.uk/modalapplication . aspx?catid=1\&pagetype=65\&appid=11\&mode=detail\&id=9012

[2] Della Vecchia, P., Malgieri, D., Nicolosi, F., and De Marco, A., "Numerical analysis of propeller effects on wing aerodynamic: tip mounted and distributed propulsion," Transportation research procedia, Vol. 29, 2018, pp. 106-115. 
[3] Patterson, M. D., Daskilewicz, M. J., and German, B., "Simplified Aerodynamics Models to Predict the Effects of Upstream Propellers on Wing Lift," 53rd AIAA Aerospace Sciences Meeting, 2015, p. 1673.

[4] Deere, K. A., Viken, J. K., Viken, S., Carter, M. B., Wiese, M., and Farr, N., "Computational analysis of a wing designed for the X-57 distributed electric propulsion aircraft," 35th AIAA applied aerodynamics conference, 2017, p. 3923.

[5] Stoll, A. M., and Veble Mikic, G., "Design studies of thin-haul commuter aircraft with distributed electric propulsion," 16th AIAA Aviation Technology, Integration, and Operations Conference, 2016, p. 3765.

[6] Rizzi, S. A., Palumbo, D. L., Rathsam, J., Christian, A. W., and Rafaelof, M., "Annoyance to noise produced by a distributed electric propulsion high-lift system," 23rd AIAA/CEAS Aeroacoustics Conference, 2017, p. 4050.

[7] Nark, D. M., Jones, W. T., Buning, P. G., and Derlaga, J. M., "High-Lift Propeller Noise Prediction for a Distributed Electric Propulsion Flight Demonstrator," 23rd AIAA/CEAS Aeroacoustics Conference, 2017, p. 3713.

[8] Pascioni, K., and Rizzi, S. A., "Tonal Noise Prediction of a Distributed Propulsion Unmanned Aerial Vehicle," 2018 AIAA/CEAS Aeroacoustics Conference, 2018, p. 2951.

[9] Pascioni, K. A., Rizzi, S. A., and Schiller, N., "Noise Reduction Potential of Phase Control for Distributed Propulsion Vehicles," AIAA Scitech 2019 Forum, 2019, p. 1069.

[10] Ashcraft, S. W., Padron, A. S., Pascioni, K. A., Stout Jr, G. W., and Huff, D. L., "Review of propulsion technologies for N+ 3 subsonic vehicle concepts," 2011.

[11] Synodinos, A., Self, R., and Torija, A., "Preliminary noise assessment of aircraft with distributed electric propulsion," 2018 AIAA/CEAS Aeroacoustics Conference, 2018, p. 2817.

[12] Kim, H. D., Perry, A. T., and Ansell, P. J., "A review of distributed electric propulsion concepts for air vehicle technology," 2018 AIAA/IEEE Electric Aircraft Technologies Symposium (EATS), IEEE, 2018, pp. 1-21.

[13] Vos, R., "Preliminary sizing of hybrid electric aircraft - Part I: Powertrain design." Aircraft Electric Propulsion, Vol. VKI LS 2019-02, von Karman Institute, Rhode-St-Genèse, 2019.

[14] Fink, M. R., “Approximate prediction of airframe noise,” Journal of Aircraft, Vol. 13, No. 11, 1976, pp. $833-834$.

[15] Huff, D. L., Henderson, B. S., and Envia, E., "Motor noise for electric powered aircraft," 22nd AIAA/CEAS Aeroacoustics Conference, 2016, p. 2882.

[16] Posey, J., Tinetti, A., and Dunn, M., "The low-noise potential of distributed propulsion on a catamaran aircraft," 12 th AIAA/CEAS Aeroacoustics Conference (27th AIAA Aeroacoustics Conference), 2006, p. 2622.

[17] Cadence NUMECA, "FINE/Open with OpenLabs 10.1 User's Guide,”, 2021. URL https://portal.numeca.be/docs/ Default.htm\#FINE0pen/Resources/PDF/FINEOpen-User-Guide.pdf

[18] Platt, R. C., “Turbulence Factors of NACA Wind Tunnels as Determined by Sphere Tests,”, 1936.

[19] Roger, M., "Aeroacoustics of installed propellers," Aircraft Electric Propulsion, Vol. VKI LS 2019-02, von Karman Institute, Rhode-St-Genèse, 2019.

[20] Amiet, R. K., "Noise due to Turbulent Flow past a Trailing Edge,” Journal of Sound and Vibration, Vol. 47, No. 3, 1976, pp. 387-393.

[21] Roger, M., and Moreau, S., "Back-Scattering Correction and Further Extensions of Amiet's Trailing-Edge Noise Model. Part 1: Theory," Journal of Sound and Vibration, Vol. 286, 2005, pp. 477-506.

[22] Glegg, S., and Devenport, W., Aeroacoustics of low Mach number flows: fundamentals, analysis, and measurement, Academic Press, 2017.

[23] Carolus, T., Schneider, M., and Reese, H., "Axial flow fan broad-band noise and prediction," Journal of Sound and Vibration, Vol. 300, No. 1, 2007, pp. 50 - 70.

[24] Shur, M., Strelets, M., Travin, A., Christophe, J., Kucukcoskun, K., Schram, C. F., Sack, S., and Åbom, M., "Effect of Inlet Distortions on Ducted Fan Noise," 22nd AIAA/CEAS Aeroacoustics Conference, ???? https://doi.org/10.2514/6.2016-2819. URL https://arc.aiaa.org/doi/abs/10.2514/6.2016-2819. 
[25] Casalino, D., Hazir, A., and Mann, A., "Turbofan Broadband Noise Prediction Using the Lattice Boltzmann Method," AIAA Journal, Vol. 56, No. 2, 2018, pp. 609-628. https://doi.org/10.2514/1.J055674

[26] Ffowcs Williams, J. E., and Hawkings, D. L., "Sound generation by turbulence and surfaces in arbitrary motion," Philosophical Transactions of the Royal Society of London. Series A, Mathematical and Physical Sciences, Vol. 264, No. 1151, 1969, pp. 321-342.

[27] Mudry, M., "La theorie generale des nappes et filaments tourbillonnaires et ses applications a l'aerodynamique instationnaire," Ph.D. thesis, PhD Thesis, Université Paris VI, 1982.

[28] Prieur, J., and Rahier, G., “Aeroacoustic integral methods, formulation and efficient numerical implementation," Aerospace Science and Technology, Vol. 5, No. 7, 2001, pp. 457-468.

[29] Rahier, G., Huet, M., and Prieur, J., "Additional terms for the use of Ffowcs Williams and Hawkings surface integrals in turbulent flows," Computers \& Fluids, Vol. 120, 2015, pp. 158-172.

[30] Juvigny, X., “A Fast Algebraic Boundary Integral Solver,” Venise: ECCOMAS paper, 1998.

[31] Amiet, R. K., “Acoustic Radiation from an Airfoil in a Turbulent Stream,” Journal of Sound and Vibration, Vol. 41, No. 4, 1975, pp. 407-420.

[32] Paterson, R. W., and Amiet, R. K., "Noise and Surface Pressure Response of an Airfoil to Incident Turbulence," J. Aircraft, Vol. 14, No. 8, 1977, pp. 729-736.

[33] De Santana, L., Christophe, J., Schram, C., and Desmet, W., "A rapid distortion theory modified turbulence spectra for semi-analytical airfoil noise prediction," Journal of sound and vibration, Vol. 383, 2016, pp. 349-363. https://doi.org/10.1016/j. jsv.2016.07.026

[34] Roger, M., Schram, C., and Santana, L. D., "Reduction of Airfoil Turbulence-Impingement Noise by Means of Leading-Edge Serrations and/or Porous Material," 19th AIAA/CEAS Aeroacoustics Conference, ???? https://doi.org/10.2514/6.2013-2108. URL https://arc.aiaa.org/doi/abs/10.2514/6.2013-2108

[35] Lyu, B., Azarpeyvand, M., and Sinayoko, S., "Prediction of noise from serrated trailing edges," Journal of Fluid Mechanics, Vol. 793, 2016, p. 556-588. https://doi.org/10.1017/jfm.2016.132

[36] Lee, S., "Empirical Wall-Pressure Spectral Modeling for Zero and Adverse Pressure Gradient Flows," AIAA Journal, Vol. 56, No. 5, 2018, pp. 1818-1829. https://doi.org/10.2514/1.J056528.

[37] Kucukcoskun, K., Christophe, J., Schram, C., and Tournour, M., "Broadband Scattering of the Turbulence-Interaction Noise of a Stationary Airfoil: Experimental Validation of the Semi-Analytical Model," Intl. Jou. of Aeroacoustics, Vol. 1\&2, 2013, pp. 85-104.

[38] Curle, N., "The Influence of Solid Boundaries upon Aerodynamic Sound," Proceedings of the Royal Society of London. Series A, Mathematical and Physical Sciences, Vol. 231, No. 1187, 1955, pp. 505-514.

[39] Schwartzschild, K., "Die Beugung und Polarisation des Lichts durch einen Spalt - i," Mathematische Annalen, Vol. 55, 1902, pp. 177-247.

[40] Abramowitz, M., and Stegun, I. A., Handbook of Mathematical Functions with Formulas, Graphs, and Mathematical Tables, Courier Dover Publications, 1964.

[41] Christophe, J., Kucukcoskun, K., Schram, C., Idier, A., Laffay, P., and Moreau, S., "Experimental Validation of a Semi-Analytical Trailing-Edge Noise Model Including Broadband Scattering," AIAA Aviation 2015, 2015.

[42] Schlinker, R. H., and Amiet, R. K., “Helicopted Rotor Trailing Edge Noise,”, 1981. CR 3470.

[43] Sinayoko, S., Kingan, M., and Agarwal, A., "Trailing edge noise theory for rotating blades in uniform flow," Proc. R. Soc. A, Vol. 469, 2013, p. 20130065.

[44] Christophe, J., Sanjosé, M., and Moreau, S., "Uncertainty Quantification of a Low-Speed Axial Fan Self-Noise," Proceedings ISROMAC Conference 2012, 2012.

[45] Siemens PLM Industry, “Simcenter 3D Acoustics User's Guide,", 2021. URL https://docs.plm.automation.siemens.com/tdoc/ scnastran/2020_1/help/\#uid:index 
[46] von Estorff, O., Boundary Elements in Acoustics, WIT Press, 2000.

[47] Kucukcoskun, K., Christophe, J., Anthoine, J., Schram, C., and Tournour, M., “An Extension of Amiet's Theory for SpanwiseVarying Incident Turbulence Noise and Broadband Scattering Using BEM," 16th AIAA/CEAS Aeroacoustics Conference, ???? https://doi.org/10.2514/6.2010-3987. URL https://arc.aiaa.org/doi/abs/10.2514/6.2010-3987

[48] Gerard, F., Tournour, M., Masri, N. E., Cremers, L., Felice, M., and Selmane, A., "Numerical Modeling of Engine Noise Radiation Through the Use of Acoustic Transfer Vectors: A Case Study," SAE paper, 2002. 2001-01-1514.

[49] Kucukcoskun, K., "Prediction of Free and Scattered Acoustic Fields of Low-Speed Fans," Ph.D. thesis, École Centrale Lyon, 2012.

[50] Bériot, H., Prinn, A., and Gabard, G., "Efficient implementation of high-order finite elements for Helmholtz problems," International Journal for Numerical Methods in Engineering, Vol. 106, No. 3, 2016, pp. 213-240.

[51] Bériot, H., and Gabard, G., "Anisotropic adaptivity of the p-FEM for time-harmonic acoustic wave propagation," Journal of Computational Physics, Vol. 378, 2019, pp. 234-256.

[52] Bériot, H., and Modave, A., "An automatic perfectly matched layer for acoustic finite element simulations in convex domains of general shape," International Journal for Numerical Methods in Engineering, Vol. 122, No. 5, 2021, pp. 1239-1261.

[53] Cadence NUMECA, "FINE/Turbo 15.1 User's Guide,", 2021. URL https://portal.numeca.be/docs/Default.htm\#FINETurbo/ Resources/PDF/FINETurbo\%20User\%20Guide.pdf

[54] He, L., and Ning, W., "Efficient Approach for Analysis of Unsteady Viscous Flows in Turbomachines," AIAA Journal, Vol. 36, No. 11, 1998, pp. 2005-2011. https://doi.org/10.2514/2.328.

[55] Roger, M., "On combined propeller synchronization and edge scattering for the noise reduction of distributed propulsion systems," 26th International Congress on Sound and Vibration, 2019.

[56] Sinnige, T., Ragni, D., Malgoezar, A. M., Eitelberg, G., and Veldhuis, L. L., "APIAN-INF: an aerodynamic and aeroacoustic investigation of pylon-interaction effects for pusher propellers," CEAS Aeronautical Journal, Vol. 9, No. 2, 2018, pp. 291-306.

[57] Rienstra, S., and Hirschberg, A., An Introduction to Acoustics, 2021. URL https://www.win.tue.nl/ sjoerdr/papers/boek.pdf

[58] Morse, P. M., and Ingard, K. U., Theoretical acoustics, McGraw-Hill Book Company, 1968. 